\title{
Non-Abelian discrete gauge symmetries in F-theory
}

\author{
Thomas W. Grimm, Tom G. Pugh and Diego Regalado \\ Max Planck Institute for Physics, \\ Föhringer Ring 6, 80805 Munich, Germany \\ E-mail: grimm@mpp.mpg.de, pught@mpp.mpg.de, regalado@mpp.mpg.de
}

ABSTRACT: The presence of non-Abelian discrete gauge symmetries in four-dimensional F-theory compactifications is investigated. Such symmetries are shown to arise from sevenbrane configurations in genuine F-theory settings without a weak string coupling description. Gauge fields on mutually non-local seven-branes are argued to gauge both $\mathrm{R}-\mathrm{R}$ and NS-NS two-form bulk axions. The gauging is completed into a generalisation of the Heisenberg group with either additional seven-brane gauge fields or R-R bulk gauge fields. The former case relies on having seven-brane fluxes, while the latter case requires torsion cohomology and is analysed in detail through the M-theory dual. Remarkably, the M-theory reduction yields an Abelian theory that becomes non-Abelian when translated into the correct duality frame to perform the F-theory limit. The reduction shows that the gauge coupling function depends on the gauged scalars and transforms non-trivially as required for the groups encountered. This field dependence agrees with the expectations for the kinetic mixing of seven-branes and is unchanged if the gaugings are absent.

KEYwords: F-Theory, Discrete and Finite Symmetries, Supersymmetric Effective Theories

ArXiv ePrint: 1504.06272 


\section{Contents}

1 Introduction 1

2 Non-Abelian discrete symmetries in four dimensions 4

2.1 Non-Abelian discrete symmetries 4

2.2 Supersymmetric non-Abelian gaugings and non-minimal kinetic terms 5

3 Non-Abelian discrete symmetries in Type IIB orientifolds $\quad 6$

3.1 Heisenberg isometries in Type IIB orientifold compactifications $\quad 7$

3.2 Non-Abelian gaugings from Type IIB orientifolds with torsion 8

4 Non-Abelian discrete symmetries in F-theory 11

4.1 Heisenberg symmetries in non-perturbative Type IIB 11

$\begin{array}{lll}4.2 & \text { Non-Abelian gaugings from seven-branes - origins } & 14\end{array}$

4.3 Non-Abelian gaugings from seven-branes - gauge invariant structures $\quad 16$

5 Non-Abelian discrete symmetries via F-/M-theory duality 19

$\begin{array}{lll}5.1 & \text { Non-harmonic reduction of M-theory } & 19\end{array}$

5.2 Dualisation of the M-theory effective action 22

6 Conclusions $\quad 25$

$\begin{array}{ll}\text { A Dualisation of the three-dimensional action } & 27\end{array}$

\section{Introduction}

In recent years phenomenological aspects of F-theory compactifications [1] have been considered intensively. While a complete understanding of the effective actions arising in such compactifications is still lacking there has been major progress investigating core aspects of the theories that arise. Much of these efforts have focused on uncovering the geometric manifestation of symmetries of the effective theories in F-theory. For example, a detailed picture of the local continuous non-Abelian and Abelian gauge symmetries has started to emerge. A state-of-the-art discussion on non-Abelian symmetries in F-theory can be found in [2-5], while recent results on Abelian gauge symmetries are found in [6-21]. The investigation of discrete symmetries in F-theory has only recently attracted more attention [17, 22-28]. The class of discrete symmetries are thereby realised as low energy remnants of Abelian gauge symmetries that are massive even in the absence of any flux background. This makes these symmetries necessarily Abelian. In this work we aim to generalize the results of $[24,25,27]$ and discuss the appearance of non-Abelian discrete symmetries in F-theory. 
In F-theory various aspects of the physics of intersecting seven-branes are captured by higher-dimensional two-torus fibered geometries. It turns out, however, that extracting the low energy implications of a given geometry is a challenging task. This can be traced back to the fact, that there is no known twelve-dimensional formulation of F-theory and the theory has to be studied either by a generalised Type IIB string perspective or by performing a duality to M-theory. Approaching F-theory directly from the Type IIB perspective seems to avoid the use of any dualities. However, as of now the correct global treatment of intersecting seven-branes is poorly understood and can be very involved, even in simple higher-dimensional compactifications. This is particularly apparent when dealing with two or more seven-branes that are mutually non-local, i.e. they cannot be rotated to D7-branes by the $S l(2, \mathbb{Z})$ symmetry at the same time. ${ }^{1}$ As we will find in this work, this is precisely the kind of brane configurations that can realise certain non-Abelian discrete symmetries. Such situations are better understood using the duality to M-theory. In this case, however, one also has to face a major complication. Since generating global discrete symmetries in a theory of quantum gravity requires that these are obtained from broken local gauge symmetries (see, e.g. [30]), one typically has to have a proper treatment of massive states in the effective action. This can be involved when using the M-theory to F-theory limit, since one needs to disentangle whether a mass of a state is actually present in F-theory or is a remnant of the fact that the F-theory limit has not been performed.

A key example of the complications which arise when dealing with massive modes in F-theory is given by so-called 'geometrically massive' U(1) gauge symmetries discussed in $[6,31-33]$. Such massive U(1)s are familiar from Type IIB orientifold compactifications, where they arise from specific configurations of D7-branes and their orientifold images [34]. The brane $\mathrm{U}(1) \mathrm{s}$ are massive even in the absence of brane fluxes with a mass proportional to the string coupling. Leaving the Type IIB weak string coupling limit requires one to realise such massive U(1)s via a torus-fibered geometry used in M-theory. Geometrically massive $\mathrm{U}(1) \mathrm{s}$ are then believed to arise from the expansion into non-harmonic forms. These forms might be described by non-trivial torsion in cohomology [27, 31, 35, 36], but eventually require the M-theory geometries considered to be extended to include non-Kähler spaces [6, 31-33]. Remarkably, this allows these U(1)s to mix also with the Kaluza-Klein vector used in connecting F-theory and M-theory. It was argued in $[24,25,27]$ that this is the proper interpretation of the physics induced on certain torus-fibered geometries with multisection. Such massive U(1)s were argued to lead to interesting discrete Abelian symmetries restricting, for example, the Yukawa couplings of the effective theories [25, 26, 37].

Given the success of identifying at least certain discrete Abelian symmetries in the F-theory geometry one might hope to be able to straightforwardly generalize the setting to the non-Abelian case. However, this leads immediately to some obstacles. Firstly, the study of geometries with multi-section seems to suggest that only Abelian symmetries naturally appear in such settings. ${ }^{2}$ Secondly, as we will see in more detail below, including non-closed forms in the reduction of M-theory accessed via eleven-dimensional supergravity seemingly

\footnotetext{
${ }^{1} \mathrm{~A}$ recent example of this fact is analysed in [29].

${ }^{2}$ Indeed, it has been proposed that the Tate-Shafarevich group determines the discrete symmetries arising in F-theory [22]. This group, however, is always Abelian and therefore obscures any generalisation.
} 
only yields Abelian gaugings. How can a non-Abelian discrete symmetry ever arise? This appears particularly puzzling, since we know from the analysis of the Type IIB supergravity actions that non-Abelian discrete symmetries actually do occur in reductions with nonclosed forms representing torsion cohomology [36, 38]. These arise from gauging a subgroup of the isometry group of the moduli space and are known to span a generalisation of the Heisenberg group. In this work we resolve these puzzles by explaining that they can be traced back to the fact that the M-theory reduction is performed in an inconvenient duality frame working with the M-theory three-form $C_{3}$ only. While the gaugings in the M-theory reduction with $C_{3}$ appear to be Abelian they actually dualise into non-Abelian gaugings of the Heisenberg algebra in the duality frame required to perform the F-theory limit.

Our findings admit an interesting Type IIB interpretation using the geometric Stückelberg mechanism. Recall that on a D7-brane this mechanism only allows one to gauge the R-R two-form axion with the brane $\mathrm{U}(1)$. This implies that the $S l(2, \mathbb{Z})$-images generally allow for a gauging of the NS-NS two-form axions as well. If one now includes two seven-branes that are mutually non-local we will show that this can imply that a third vector has to complete the gauging into a non-Abelian group. This additional vector can arise either from the R-R bulk sector, in which case the gaugings are purely geometrical, or from another seven-brane, in which case brane fluxes are required. We argue that the former possibility admits a direct interpretation in the M-theory fourfold geometry. The non-Abelian completion of the gauging turns out to be a consistency condition on the compact fourfold when considering non-closed forms. It admits a natural mathematical interpretation in terms of torsion cohomology for Calabi-Yau fourfolds. Let us stress that while we believe the required fourfolds with the appropriate torsion cohomology exist, we will not attempt to find an explicit example but rather study the general implications.

The non-Abelian gauge groups that we find are shown to be generalisations of the Heisenberg group. The fact that these groups are neither semisimple nor compact has important consequences on the form of the effective action. It implies that the gauge coupling function of this group cannot be simply constant, since there exists no positive definite Killing form for these groups. It instead has to be a non-trivial holomorphic function of the complex scalars transforming under the gauge group. Interestingly the gauge coupling function is independent of the constants determining the gauged subalgebra of the isometry group. This implies that they are present for any gauging and we argue that they can be used to determine the allowed non-Abelian gauge algebra.

The paper is organised as follows. In section 2 we review how non-Abelian discrete symmetries can arise as gaugings of isometries in four dimensions. The Type IIB string theory embedding of a special type of gaugings is discussed in section 3 . We show that when using seven-brane gauge fields to yield such gaugings the introduction of mutually non-local seven-branes is crucial. This suggests that a proper treatment should invoke an F-theory geometry and the duality to M-theory. The F-theory setting and the allowed gaugings are discussed in section 4, while the explicit M-theory reduction is then performed in section 5. We show that the Abelian gaugings dualize to non-Abelian gaugings upon changing to the duality frame that allows the F-theory limit to be performed. Details on the computations are supplemented in appendix A. 


\section{Non-Abelian discrete symmetries in four dimensions}

In this section we briefly review the realisation of discrete gauge symmetries in field theory $[30,36,39]$. We also include a discussion of supersymmetry and comment on the structure of non-minimal gauge-kinetic terms for non-Abelian groups that are neither semisimple nor compact.

\subsection{Non-Abelian discrete symmetries}

By discrete gauge symmetry we simply mean a discrete remnant of a spontaneously broken gauge symmetry. Let us consider the simplest Abelian example to illustrate this, namely the Stückelberg Lagrangian for a vector $A$ and a scalar $\phi$ of periodicity $2 \pi$,

$$
\mathcal{L}=-\frac{1}{2 g^{2}} d A \wedge * d A-\mu^{2}(d \phi-k A) \wedge *(d \phi-k A),
$$

where $g$ is the YM coupling constant, $\mu$ is a mass scale and $k \in \mathbb{Z}$. This Lagrangian is invariant under the local transformations

$$
\delta A=d \lambda, \quad \delta \phi=k \lambda
$$

and we find that the space of physically distinct vacua is given by $A=0$ and $\phi=\phi_{0}$ with $\phi_{0}$ a constant in $[0,2 \pi)$. Then, we immediately see that this system breaks the underlying $\mathrm{U}(1)$ symmetry since under a constant gauge transformation we find that the vacuum is not invariant. Indeed, if we consider the vacuum defined by $\left|\phi_{0}\right\rangle$, then after such gauge transformation we arrive at $\left|\phi_{0}+k \lambda\right\rangle$, which is in general different from $\left|\phi_{0}\right\rangle$. However, due to the presence of the integer $k$ and the periodicity of $\phi$, we may still find non-trivial gauge transformations that preserve the vacuum, namely $\lambda=\frac{2 \pi}{k}$, which form a $\mathbb{Z}_{k}$ subgroup of $\mathrm{U}(1)$ parameterised by $e^{i \lambda}$. Let us stress that the fluctuations of the vector $A$ around these vacua is massive with mass $k^{2} \mu^{2}$. This implies that an effective theory arising from string theory has to include massive modes.

As shown in [36], in order to generalise this to non-Abelian discrete symmetries, it proves useful to think of (2.1) as the gauging of a scalar manifold with a U(1) isometry with charge $k$. In that case we start with a scalar manifold $S^{1}$, whose isometry group is generated by $t=\partial_{\phi}$. Furthermore, the particular gauging we consider is related to picking a Killing vector with the following normalisation

$$
X=k \partial_{\phi} .
$$

Then, the orbit associated to $X$ is a map $Q: S^{1} \times \mathrm{U}(1) \rightarrow S^{1}$ that takes a point $\phi_{0} \in S^{1}$ and the element $e^{i \lambda} \in \mathrm{U}(1)$ to give $Q\left(\phi_{0} ; \lambda\right)=\phi_{0}+k \lambda$. Then, we see that for a given vacuum $\phi_{0}$, the subgroup that is not broken corresponds to the solutions to $Q\left(\phi_{0} ; \lambda\right)=\phi_{0}$ which again leads to $\mathbb{Z}_{k}$. Notice that the discrete symmetry is encoded in the relative normalisation of the Killing vector (2.3) with respect to the gauge algebra generator.

Next we discuss the gauging of non-Abelian isometries with the appropriate charges. Consider a sigma model with a $d$-dimensional manifold $\mathcal{M}$ endowed with a Riemannian metric $g$ and coordinates $\phi^{a}$,

$$
\mathcal{L}_{0}=-g_{a b} d \phi^{a} \wedge * d \phi^{b},
$$


and let $t_{\hat{A}}$ be generators of the group of isometries $\operatorname{Iso}(\mathcal{M})$ which satisfy

$$
\left[t_{\hat{A}}, t_{\hat{B}}\right]=f_{\hat{A} \hat{B}} \hat{C} t_{\hat{C}}
$$

where $f_{\hat{A} \hat{B}} \hat{C}$ are the structure constants. A particular gauging is specified by picking a set of Killing vectors

$$
X_{A}=k_{A}^{\hat{A}} t_{\hat{A}}
$$

where $k_{A}^{\hat{A}}$ are constants and the vectors generate the gauge algebra

$$
\left[X_{A}, X_{B}\right]=f_{A B}^{C} X_{C}
$$

The gauging is implemented by considering the following Lagrangian

$$
\mathcal{L}=-\frac{1}{2} f_{A B}^{1} F^{A} \wedge * F^{B}-\frac{1}{2} f_{A B}^{2} F^{A} \wedge F^{B}-g_{a b} D \phi^{a} \wedge * D \phi^{b},
$$

where we included gauge bosons $A^{B}$ with field strengths $F^{B}=d A^{B}+f_{A C}{ }^{B} A^{A} \wedge A^{C}$. The functions $f_{A B}^{1}$ and $f_{A B}^{2}$ are in general dependent on the scalars $\phi^{a}$. $f_{A B}^{1}$ determines the gauge couplings and has to be positive definite. We stress that $f_{A B}^{i}, i=1,2$ in general have to transform non-trivially under the gauge group in order to ensure gauge invariance of the Lagrangian, i.e. one has to have

$$
\delta f_{A B}^{i}=\lambda^{C}\left(f_{C A}^{D} f_{B D}^{i}+f_{C B}^{D} f_{A D}^{i}\right),
$$

where $\lambda^{D}$ are the gauge parameters. In particular, for groups that are noncompact one cannot use the Killing form and therefore $f_{A B}^{1}$ and $f_{A B}^{2}$ have to be non-trivial functions of the fields $\phi^{a}$. Furthermore, we defined the covariant derivatives

$$
D \phi^{a}=d \phi^{a}-A^{B} X_{B}^{a}
$$

Now we may proceed formally in analogy to the Abelian case. The space of inequivalent vacua of the gauged theory (2.8) is $A^{B}=0$ and constant $\phi^{a} \in \mathcal{M}$. Then, under a constant gauge transformation along $\lambda^{A} X_{A}$ we find that the vacuum $\phi_{0}^{a}$ goes to $Q\left(\phi_{0}^{a} ; \lambda^{A}\right)$ which, if different from $\phi_{0}^{a}$, signals a spontaneous breaking of the generator $\lambda^{A} X_{A}$. Alternatively, the set of $e^{i \lambda^{A} X_{A}}$ that satisfy $Q\left(\phi_{0}^{a} ; \lambda^{A}\right)=\phi_{0}^{a}$ corresponds to a preserved symmetry. Clearly, this construction may lead to a case in which the preserved symmetry is a non-Abelian discrete subgroup of $\operatorname{Iso}(\mathcal{M})$. In the following section we consider a particular example in which $\operatorname{Iso}(\mathcal{M})$ is a generalisation of the Heisenberg group.

\subsection{Supersymmetric non-Abelian gaugings and non-minimal kinetic terms}

Up to now we did not discuss the supersymmetric version of the above setting. In order to do that one has to first realise that four-dimensional $\mathcal{N}=1$ supersymmetry implies that $\mathcal{M}$ is a Kähler manifold. We denote the complex coordinates by $\Phi^{I}$. The couplings $f_{A B}^{1}$ 
and $f_{A B}^{2}$ have to combine into a function $f_{A B}=f_{A B}^{1}+i f_{A B}^{2}$ that is holomorphic in the $\Phi^{I}$. The bosonic part of a supersymmetric Lagrangian will then include the terms

$$
\mathcal{L}=-\frac{1}{2} \operatorname{Re} f_{A B} F^{A} \wedge * F^{B}-\frac{1}{2} \operatorname{Im} f_{A B} F^{A} \wedge F^{B}-K_{I \bar{J}} D \Phi^{I} \wedge * D \bar{\Phi}^{J}-V * 1,
$$

where $K_{I \bar{J}}=\partial_{\Phi^{I}} \partial_{\bar{\Phi}^{J}} K$ is locally the derivative of a Kähler potential $K$. Crucially the isometries that can now be gauged have to be holomorphic such that

$$
\delta A^{A}=d \Lambda^{A}+f_{B C}{ }^{A} A^{B} \Lambda^{C} \quad \delta \Phi^{I}=\Lambda^{A} X_{A}^{I}(\Phi)
$$

which induces the transformation (2.9).

Let us stress that in general one has to impose additional conditions on gaugings allowed by $\mathcal{N}=1$ supersymmetry. Consider, for example, the simple Kähler potential $K=\frac{1}{2}(\phi+\bar{\phi})^{2}$, which yields a constant Kähler metric and a Lagrangian that admits the shift symmetry $\phi \rightarrow \phi+\lambda_{1}+i \lambda_{2}$ with real constants $\lambda_{i}$. Supersymmetry implies that the two shift symmetries labelled by $\lambda_{i}$ cannot be gauged by different $\mathrm{U}(1)$ vectors $A^{i}$, since the D-term scalar potential would not be gauge-invariant. In our examples the situation will be even more subtle. Since the gaugings also have to be compatible with the holomorphicity of the gauge coupling function even if there exists a gauge-invariant scalar potential.

If the isometry group $\operatorname{Iso}(\mathcal{M})$ that we want to gauge is semisimple and compact, we may take $f_{A B}$ to be a holomorphic function of the ungauged scalars proportional to the Killing form. In this case $f_{A B}$ satisfies the constraints imposed by supersymmetry. This implies that one can also add the kinetic terms for $A^{A}$ to the Lagrangian without having any gauged scalars. However, the isometry group need not be compact nor semisimple in which case we might be forced to include non-minimal kinetic terms for the vectors. In such cases, holomorphicity of the gauge kinetic function imposes non-trivial constraints [40].

Let us close this section with recalling yet another important issue related to the gauge-transformation of the gauge coupling function. If the four-dimensional $\mathcal{N}=1$ theory contains chiral fermions charged under an Abelian gauge symmetry, it might be necessary to employ a Green-Schwarz mechanism to chancel the one-loop anomaly induced by these fields $[41,42]$. The classical terms $\frac{1}{2} \operatorname{Im} f_{A B} F^{A} \wedge F^{B}$ are then allowed to be non-gauge invariant and fixed to induce tree-level diagrams that cancel the one-loop anomalous diagrams of the chiral fermions. In consistent string theory compactifications this mechanism is automatically implemented in the situations that require such a cancellation.

\section{Non-Abelian discrete symmetries in Type IIB orientifolds}

In this section we study the possibility of obtaining non-Abelian discrete symmetries by gauging R-R and NS-NS scalars in Type IIB orientifolds with O7-planes. We first examine the symmetries of the orientifold moduli space in subsection 3.1. The Heisenberg isometry group that appears is a special version of the symmetry groups later encountered in the complete F-theory setting. We then turn to the discussion of the gauging of this nonAbelian group in subsection 3.2 by performing a reduction with non-harmonic forms. It turns out that there is a tension between performing a supersymmetric orientifold quotient and the gauging of a non-Abelian group. 


\subsection{Heisenberg isometries in Type IIB orientifold compactifications}

To begin with let us consider Calabi-Yau orientifold compactifications of Type IIB with O7-planes. The effective action for the bulk fields in such compactifications contains the following terms [43]

$$
\begin{aligned}
\mathcal{L}= & -G_{\alpha \beta} d v^{\alpha} \wedge * d v^{\beta}-\frac{1}{4 \mathcal{V}^{2}} d \mathcal{V} \wedge * d \mathcal{V}+\frac{3 i v^{\alpha}}{(\tau-\bar{\tau})} \mathcal{K}_{\alpha a b}\left(d c^{a}-\tau d b^{a}\right) \wedge *\left(d c^{b}-\bar{\tau} d b^{b}\right) \\
& -\frac{G^{\alpha \beta}}{16 \mathcal{V}^{2}}\left(d \rho_{\alpha}+\frac{1}{2} \mathcal{K}_{\alpha a b}\left(b^{a} d c^{b}-c^{a} d b^{b}\right)\right) \wedge *\left(d \rho_{\beta}+\frac{1}{2} \mathcal{K}_{\beta c d}\left(b^{c} d c^{d}-c^{c} d b^{d}\right)\right)
\end{aligned}
$$

In this expression $\tau=C_{0}+i e^{-\phi}$ is the axiodilaton, $b^{a}, c^{a}, a=1, \ldots h_{-}^{1,1}$ arise from the reduction of $B_{2}$ and $C_{2}$ on harmonic orientifold-odd two-forms, and $\rho_{\alpha}, \alpha=1, \ldots, h_{+}^{1,1}$ comes from the reduction of $C_{4}$ on orientifold-even harmonic four-forms. The real scalars $v^{\alpha}$ are the deformations of the Kähler form of the underlying Calabi-Yau geometry. The intersection numbers of the Calabi-Yau manifold are given by

$$
\mathcal{K}_{\alpha \beta \gamma}=\int_{Y_{3}} \omega_{\alpha} \wedge \omega_{\beta} \wedge \omega_{\gamma}, \quad \mathcal{K}_{\alpha a b}=\int_{Y_{3}} \omega_{\alpha} \wedge \omega_{a} \wedge \omega_{b} .
$$

The first of these is related to the definition of the volume $\mathcal{V}=\frac{1}{6} \mathcal{K}_{\alpha \beta \gamma} v^{\alpha} v^{\beta} v^{\gamma}$ and the metric $G_{\alpha \beta}$. The Lagrangian defines a Kähler metric when written in the form (2.11) with a Kähler potential $K=-2 \log \mathcal{V}$ and complex coordinates

$$
G^{a}=c^{a}-\tau b^{a}, \quad T_{\alpha}=\rho_{\alpha}+\frac{1}{2(\tau-\bar{\tau})} \mathcal{K}_{\alpha a b} G^{a}(G-\bar{G})^{b}-\frac{1}{2} i \mathcal{K}_{\alpha \beta \gamma} v^{\beta} v^{\gamma} .
$$

Clearly, there will be additional moduli corresponding to the complex structure deformations and brane fields. These will suppressed in the following, since our current focus is on the identification of candidate non-Abelian symmetries in this sector of the theory. As we will see later, similar structures appear in the seven-brane sector.

One now readily checks that this Kähler metric has the following $2 h_{-}^{(1,1)}+h_{+}^{(1,1)}$ holomorphic isometries

$$
\delta G^{a}=\lambda_{1}^{a}-\tau \lambda_{2}^{a}, \quad \delta T_{\alpha}=\lambda_{\alpha}-\mathcal{K}_{\alpha a b} G^{b} \lambda_{2}^{a} .
$$

where $\lambda_{1}^{a}, \lambda_{2}^{a}, \lambda_{\alpha}$ are the real scalar gauge parameters. Using the transformations (3.4) one determines the holomorphic Killing vectors to be

$$
t_{(1, a)}=\partial_{G^{a}}, \quad t_{(2, a)}=-\tau \partial_{G^{a}}-\mathcal{K}_{\alpha a b} G^{b} \partial_{T_{\alpha}}, \quad t^{\alpha}=\partial_{T_{\alpha}} .
$$

Upon exponentiation these vectors yield the Lie group of isometries of $\mathcal{M}$, which we denote by $\operatorname{Iso}(\mathcal{M})$. The explicit algebra reads,

$$
\left[t_{(1, a)}, t_{(2, b)}\right]=-\mathcal{K}_{\alpha a b} t^{\alpha},
$$

with all other commutators vanishing. This algebra is a generalisation of the Heisenberg algebra and will be our prime example for the non-Abelian structures appearing in our 
string theory set-ups. Comparing with (2.5) this implies that the only non-vanishing nonAbelian structure constants are $f_{(1, a)(2, b)}{ }^{\alpha}=-\mathcal{K}_{\alpha a b}$. Finally, the fact that $c^{a}, b^{a}$ and $\rho_{\alpha}$ are periodic with period $2 \pi$, imposes the following identifications in the scalar manifold

$$
\begin{aligned}
& c^{a} \simeq c^{a}+2 \pi, \quad \text { and } \quad \rho_{\alpha} \simeq \rho_{\alpha}+\pi \mathcal{K}_{\alpha a b} b^{b}, \\
& b^{a} \simeq b^{a}+2 \pi, \quad \text { and } \quad \rho_{\alpha} \simeq \rho_{\alpha}-\pi \mathcal{K}_{\alpha a b} c^{b}, \\
& \rho_{\alpha} \simeq \rho_{\alpha}+2 \pi .
\end{aligned}
$$

These identifications render the field-space spanned by $c^{a}, b^{a}$ and $\rho_{\alpha}$ to be compact.

Let us now address the question of gauging the non-Abelian symmetries (3.6). This requires the introduction of gauge fields that arise from the bulk sector. In section 4 we will develop this further by including vectors that arise from the brane sector.

\subsection{Non-Abelian gaugings from Type IIB orientifolds with torsion}

In this section we briefly review the construction in [36] which shows that the reduction of Type IIB on manifolds $Y_{3}$ with torsion homology may lead to an effective theory where the non-Abelian isometries analysed in the last section are gauged.

In general, cohomology groups with integer coefficients are finitely generated Abelian groups, which means that they are the direct sum of cyclic groups, namely

$$
H^{p}(M, \mathbb{Z})=\underbrace{\mathbb{Z} \oplus \cdots \oplus \mathbb{Z}}_{\text {free }} \oplus \underbrace{\mathbb{Z}_{k_{1}} \oplus \cdots \oplus \mathbb{Z}_{k_{n}}}_{\text {torsion }}
$$

which, as indicated above, is the sum of a free part and a non-free (or torsion) part. The former plays a central role in string compactifications since Hodge's theorem provides an isomorphism between the free part and the space of harmonic forms, which correspond to the internal wave function of massless modes. The torsion part, however, does not yield massless modes so its role in compactifications is not as straightforward. It was argued in $[35,36]$ that including torsion forms in string reductions naturally yields discrete gauge symmetries. Also, one can obtain the correct spectrum of charged states under such discrete symmetry by wrapping different branes in the torsion homology cycles, in agreement with the expectations for a theory of quantum gravity [30].

Let us now illustrate the reduction on torsion cohomology in a simple example before moving to a more general case. We will consider the reduction of a theory with a two form potential $B_{2}$ to four dimensions on a space $M$ with torsion cohomology Tor $H^{2}(M, \mathbb{Z})=\mathbb{Z}_{k}$. Then we have a closed two-form $\Lambda_{2}$ which, in integer cohomology, is not exact but such that $k$ times $\Lambda_{2}$ is, namely

$$
k \Lambda_{2}=d \lambda_{1}, \quad d \Lambda_{2}=0
$$

for some form $\lambda_{1}$. Now if we include the torsion element $\Lambda_{2}$ in the reduction, then we must also reduce along the non-closed form $\lambda_{1}$. This follows from the fact that the Laplacian $\Delta$ commutes with the exterior derivative and from demanding that we include all the modes 
of a given mass scale. Indeed, if $\Delta \Lambda_{2}=-m^{2} \Lambda_{2}$, we should include the form $\lambda_{1}$ which satisfies $\Delta \lambda_{1}=-m^{2} \lambda_{1}$. Thus, we find that

$$
B_{2}=b \Lambda_{2}+A \wedge \lambda_{1}, \quad d B_{2}=(d b-k A) \wedge \Lambda_{2}+d A \wedge \lambda_{1}
$$

where $b$ is a four-dimensional scalar and $A$ is a four-dimensional vector. These appear in the field strength for $B_{2}$ in the combination $(d b-k A)$ which gives a Stückelberg coupling. This then leads to a theory of a massive vector with a $\mathbb{Z}_{k}$ discrete gauge symmetry.

Now we are ready to discuss the more involved case of Type IIB orientifolds with torsion. A six-dimensional manifold has only two independent torsion cohomology groups, namely

$$
\operatorname{Tor} H^{2}\left(Y_{3}\right) \simeq \operatorname{Tor} H^{5}\left(Y_{3}\right) \simeq \bigoplus_{a} \mathbb{Z}_{k_{a}}, \quad \operatorname{Tor} H^{3}\left(Y_{3}\right) \simeq \operatorname{Tor} H^{4}\left(Y_{3}\right) \simeq \bigoplus_{\alpha} \mathbb{Z}_{k_{\alpha}}
$$

where the isomorphisms follow from the universal coefficient theorem. Then, in analogy with equation (3.9) we have that ${ }^{3}$

$$
\begin{aligned}
& d \gamma_{i}=k_{i}^{a} \omega_{a}, \quad d \omega_{\alpha}=k_{\alpha \kappa} \beta^{\kappa}, \quad d \alpha_{\kappa}=k_{\alpha \kappa} \tilde{\omega}^{\alpha}, \\
& d \omega_{a}=0, \quad d \beta_{\kappa}=0, \quad d \tilde{\omega}^{\alpha}=0
\end{aligned}
$$

which are compatible with the conditions

$$
\int_{Y_{3}} \alpha_{\kappa} \wedge \beta^{\lambda}=\delta_{\kappa}^{\lambda}, \quad \int_{Y_{3}} \omega_{\alpha} \wedge \tilde{\omega}^{\beta}=\delta_{\alpha}^{\beta} .
$$

We note that in the pure torsion case the $k_{i}^{a}$ and $k_{\alpha \kappa}$ would be invertible. However, by not imposing conditions on the rank we allow harmonic and torsion forms to be considered simultaneously in the following analysis. Also, we assume that the parity under the orientifold action of $\alpha_{\kappa}, \beta^{\kappa}$ and $\omega_{\alpha}, \tilde{\omega}^{\alpha}$ is even while the parity of $\gamma_{i}$ and $\omega_{a}$ is odd.

In addition to this we will also demand that the basis of forms also satisfies

$$
\omega_{a} \wedge \gamma_{i}=M_{i a}{ }^{\kappa} \alpha_{\kappa}, \quad \omega_{a} \wedge \omega_{b}=\mathcal{K}_{\alpha a b} \tilde{\omega}^{\alpha}, \quad \gamma_{i} \wedge \gamma_{j}=N_{i j}{ }^{\alpha} \omega_{\alpha}
$$

In the first of these identities we have demanded that there is no term proportional to $\beta^{\kappa}$. This is imposed in order to prevent electric and magnetic degrees of freedom from being simultaneously gauged. The quantities $M_{i a}{ }^{\kappa}$ and $N_{i j}{ }^{\alpha}$ appearing in these identities define the additional intersection numbers

$$
M_{i a}{ }^{\kappa}=\int_{Y_{3}} \gamma_{i} \wedge \omega_{a} \wedge \beta^{\kappa}, \quad \quad N_{i j}^{\alpha}=\int_{Y_{3}} \tilde{\omega}^{\alpha} \wedge \gamma_{i} \wedge \gamma_{j} .
$$

Compatibility of these conditions then implies that

$$
k_{i}^{a} M_{j a}{ }^{\kappa}=k_{j}^{a} M_{i a}{ }^{\kappa}, \quad k_{\alpha \kappa} M_{i a}{ }^{\kappa}=k_{i}^{b} \mathcal{K}_{\alpha a b}, \quad k_{\alpha \kappa} N_{i j}{ }^{\alpha}=0 .
$$

In the second identity in (3.14), we have allowed for a non-trivial product between the torsion two-forms which, as we will see, is coupling responsible for a non-Abelian gauge symmetry.

\footnotetext{
${ }^{3}$ We did not include the torsion five-forms since we will not need them here.
} 
Given this setup, the ansatz for the reduction is

$$
\begin{aligned}
& C_{4}=V^{\kappa} \wedge \alpha_{\kappa}-U_{\kappa} \wedge \beta^{\kappa}+\rho_{\alpha} \tilde{\omega}^{\alpha}+C_{2}^{\alpha} \wedge \omega_{\alpha}, \\
& B_{2}=A^{1 i} \wedge \gamma_{i}+b^{a} \omega_{a}, \quad C_{2}=A^{2 i} \wedge \gamma_{i}+c^{a} \omega_{a} .
\end{aligned}
$$

where $C_{4}$ has an expansion into orientifold-even three-forms while $B_{2}, C_{2}$ are expanded into orientifold-odd one-forms and two-forms. Here $A^{1 i}, A^{2 i}$ and $V^{\kappa}, U_{\kappa}$ are four-dimensional vectors. Note that $V^{\kappa}$ and $U_{\kappa}$ are electric-magnetic duals by means of the self-duality of the field-strength of $C_{4}$. Similarly, the two-form $C_{2}^{\alpha}$ is the four-dimensional dual of the scalar $\rho_{\alpha}$ already used in (3.1).

The effective action which results from the ansatz (3.17) can be described in terms of the fields $C_{2}^{\alpha}$ and $U_{\kappa}$ or in terms of their duals $\rho_{\alpha}$ and $V^{\kappa}$. When working with $C_{2}^{\alpha}$ and $U_{\kappa}$ the $10 \mathrm{~d}$ field strength

$$
F_{5}=d C_{4}+\frac{1}{2}\left(B_{2} \wedge d C_{2}-C_{2} \wedge d B_{2}\right)
$$

gives rise to the four-dimensional field strengths

$$
D C_{2}^{\alpha}=d C_{2}^{\alpha}+\frac{1}{2} N_{i j}^{\alpha}\left(A^{1 i} \wedge F^{2 j}-A^{2 i} \wedge F^{1 j}\right), \quad F_{\kappa}=d U_{\kappa}-k_{\alpha \kappa} C_{2}^{\alpha},
$$

where

$$
F^{1 i}=d A^{1 i}, \quad F^{2 i}=d A^{2 i} .
$$

Here we see that the nonlinear terms in $F_{5}$ have generated a Chern-Simons modification in $D C_{2}^{\alpha}$, but that all gaugings remain Abelian.

In contrast, if one works in the dual picture and encodes all degrees of freedom by $\rho_{\alpha}$ and $V^{\kappa}$, one finds the field strengths

$$
F^{1 i}=d A^{1 i}, \quad F^{2 i}=d A^{2 i}, \quad F^{\kappa}=d V^{\kappa}+M_{i a}{ }^{\kappa} k_{j}^{a} A^{1 i} \wedge A^{2 j},
$$

where, in this dual picture, the nonlinear terms in $F_{5}$ have generated a non-Abelian structure $F^{\kappa}$. In fact, one checks by performing the reduction that the isometries of (3.4) are gauged due to the non-trivial $k_{\alpha \kappa}$ and $k_{i}^{a}$. Explicitly, the covariant derivatives read

$$
D T_{\alpha}=d T_{\alpha}+k_{\alpha \kappa} V^{\kappa}-\mathcal{K}_{\alpha a b} G^{a} k_{i}^{b} A^{1 i}, \quad D G^{a}=d G^{a}+k_{i}^{a}\left(A^{2 i}-\tau A^{1 i}\right) .
$$

This suggests that the gaugings are compatible with the holomorphic structure of the reductions. However, by performing the dimensional reduction [36] we see that the gauge coupling function derived fails to be holomorphic in the coordinates introduced above. We therefore propose that this construction is not compatible with supersymmetry without modifying the ansatz (3.12) and (3.17).

Let us add some more observations to support this further. We stress that there is a curiosity in the gaugings (3.22): for the gauged scalars $G^{a}$ it appears that the real and imaginary parts are gauged at the same time with two different vectors corresponding to noncommuting generators. We find that the Kähler potential both depends on these scalars and 
is invariant under the symmetry. This property of the gauged $G^{a}$ implies that constructing a holomorphic gauge coupling function which transforms in the appropriate fashion appears to be impossible. Furthermore we see that in the underlying $\mathcal{N}=2$ theory obtained by a Calabi-Yau reduction the fields completing $c^{a}, b^{a}$ into hypermultiplets are the scalars $\rho_{a}$ from $C_{4}$ and $v^{a}$ from the Kähler form. One can check that these scalars are ungauged and admit a scalar potential. This shows that the truncation associated with the orientifold quotient inconsistently removes the two ungauged degrees of freedom from the hypermultiplets.

We therefore find that the inclusion of torsion cohomology is by no means straightforward in the presence of an orientifold projection. It would be interesting to reveal the underlying physical reason of the incompatibility of the $\mathcal{N}=1$ orientifold truncation with the torsion proposal of [36]. Our findings suggest that torsion cohomology can only be 'straightforwardly' included for orientifold-even forms, i.e. where there are chains associated to the forms with non-vanishing physical volume. In the next subsection we will argue that when generalising the setting away form the weak string coupling limit the gaugings can be made compatible with $\mathcal{N}=1$ supersymmetry.

\section{Non-Abelian discrete symmetries in F-theory}

In this section we discuss the appearance of four-dimensional non-Abelian discrete symmetries in brane-bulk and brane-flux systems from the Type IIB perspective. This will allow us to develop different settings that naturally admit such symmetries. In order to realise these symmetries in a seven-brane sector, however, it requires the introduction of mutually non-local branes that cannot be treated at weak string coupling. To find a globally consistent description of such a system we therefore will use F-theory. While we are able to heuristically motivate our findings directly using the language of Type IIB string theory with $(p, q)$-seven-branes a more thorough justification will later, in section 5 , be given by using the M-theory approach to F-theory.

\subsection{Heisenberg symmetries in non-perturbative Type IIB}

In the previous subsection we have shown that the non-harmonic reduction yielding a nonAbelian gauge theory is not compatible with the $\mathcal{N}=1$ supersymmetry imposed by the Type IIB orientifold projection. This conflict arose from the fact that the modes arising from the fields $B_{2}$ and $C_{2}$ naturally combine into $\mathcal{N}=1$ four-dimensional scalars $G^{a}=$ $c^{a}-\tau b^{a}$ with real and imaginary parts simultaneously gauged by two different vector fields. Importantly, this analysis was a weak string coupling analysis in which the ten-dimensional $\tau$ did not vary over the internal manifold but rather descended to a four-dimensional degree of freedom. This led to the fact that the behaviour of the modes $b^{a}$ and $c^{a}$ cannot be decoupled. However, in the more general situation in which we depart from weak string coupling, the $S l(2, \mathbb{Z})$ symmetry group will have non-trivial monodromies on the compactification space and neither $\tau$ nor $c^{a}, b^{a}$ are well-defined fields in the effective theory. In the following we introduce the analogs for the fields $G^{a}$ in compactifications with varying $\tau$ and describe how the coupling to seven-branes introduces a non-Abelian gauge structure. 
Let us now work on the Kähler manifold $\mathcal{B}_{3}$, which is the base of an elliptically fibered Calabi-Yau fourfold $Y_{4}$ that we use for the F-theory treatment. In settings with weak coupling limit one has $\mathcal{B}_{3}=Y_{3} / \mathbb{Z}_{2}$. When working in Type IIB language one would need to expand

$$
C_{2}-\tau B_{2}=N^{a} \hat{\Psi}_{a},
$$

where $\hat{\Psi}_{a}$ are appropriate two-forms on an $S l(2, \mathbb{Z})$-bundle on $\mathcal{B}_{3}$ and $N^{a}$ are complex scalar fields in four dimensions. The two-forms $\hat{\Psi}_{a}$ will in general depend on the complex structure moduli of $\mathcal{B}_{3}$ and the seven-brane positions. It is expected that the explicit construction of the $\hat{\Psi}_{a}$ is challenging. However, the Calabi-Yau fourfold $Y_{4}$ turns out to be a powerful tool to capture this information in a more tractable way.

On $Y_{4}$ the information encoded in $\hat{\Psi}_{a}$ is captured by a certain basis of $(2,1)$-forms $\Psi_{a}$ that do not descend from $(2,1)$-forms of the base $\mathcal{B}_{3}$. The additional constraint is often stated as the requirement that the $\Psi_{a}$ have a component with one leg in the fiber of $Y_{4}$. In the simplest situation $\Psi_{a}$ are harmonic forms that are parameterising $H^{2,1}\left(Y_{4}\right)$ but are not elements of $H^{2,1}\left(\mathcal{B}_{3}\right)$. In the following, we will first consider only harmonic $(2,1)$-forms, but later generalise to include non-closed and exact forms. To obtain the four-dimensional fields $N^{a}$ in (4.1) one now has to expand a three-form $C_{3}$ into the (2,1)-forms $\Psi_{a}$ as follows

$$
C_{3}=N^{a} \Psi_{a}+\bar{N}^{a} \bar{\Psi}_{a}+\ldots
$$

This is motivated by the M-theory to F-theory limit as we discuss in section 5 . In this limit the $N^{a}$ lift to four-dimensional scalars that include the scalars coming from $B_{2}, C_{2}$. Furthermore, despite the abuse of notation for the indices, the $N^{a}$ will also contain the seven-brane Wilson lines. To display the effective action one first has to gain some deeper understanding of the moduli dependence of $\Psi_{a}$. Clearly, since these are $(2,1)$-forms on $Y_{4}$ they will vary with the complex structure moduli of $Y_{4}$. For $H^{2,1}\left(Y_{4}\right)$ one can in fact argue that the $\Psi_{a}$ admit an expansion

$$
\Psi_{a}=\frac{1}{2} \operatorname{Re} f_{a b}\left(\beta^{b}-i \bar{f}^{b c} \alpha_{c}\right), \quad \Psi_{a}-\bar{\Psi}_{a}=-i \alpha_{a},
$$

where $\left(\alpha_{a}, \beta^{a}\right)$ are a real three-form basis for the elements of $H^{3}\left(Y_{4}\right)$ which are not in $H^{2,1}\left(\mathcal{B}_{3}\right)$ and $f^{a b}$ is a holomorphic function of the complex structure moduli. The $\Psi_{a}$ are not anti-holomorphic in the complex structure moduli due to the appearance of $\operatorname{Re} f_{a b}$, the inverse of $\operatorname{Re} f^{a b}$. Using the real basis $\left(\alpha_{a}, \beta^{a}\right)$ we can also expand

$$
C_{3}=a^{a} \alpha_{a}-b_{a} \beta^{a}+\ldots
$$

where $\left(a^{a}, b_{a}\right)$ are real scalars. Comparing (4.2) with (4.4) and using (4.3), we see that

$$
N^{a}=-i\left(a^{a}+i f^{a b} b_{b}\right) .
$$

In a next step we recall the effective action for the complex scalars $N^{a}$ coupled to $v^{\alpha}, \rho_{\alpha}$ and study its symmetries. The derivation of this action proceeds via M-theory as carried out in [44]. This yields the generalisation of the weak string coupling action (3.1) to F-theory as 


$$
\begin{aligned}
\mathcal{L}= & -G_{\alpha \beta} d v^{\alpha} \wedge * d v^{\beta}-\frac{1}{4 \mathcal{V}^{2}} d \mathcal{V} \wedge * d \mathcal{V}+\frac{3 v^{\alpha}}{\mathcal{K}} d_{\alpha a b} d N^{a} \wedge * d \bar{N}^{b} \\
& -\frac{G^{\alpha \beta}}{16 \mathcal{V}^{2}}\left(d \rho_{\alpha}+\frac{i}{2}\left(d_{\alpha a c} \bar{N}^{c} d N^{a}-d_{\alpha c a} N^{c} d \bar{N}^{a}\right)\right) \wedge *\left(d \rho_{\beta}+\frac{i}{2}\left(d_{\beta b d} \bar{N}^{d} d N^{b}-d_{\beta d b} N^{d} d \bar{N}^{b}\right)\right)
\end{aligned}
$$

with

$$
d_{\alpha a b}=i \int_{Y_{4}} \omega_{\alpha} \wedge \Psi_{a} \wedge \bar{\Psi}_{b} .
$$

Here $\omega_{\alpha}$ is a two-form dual to vertical divisors $D_{\alpha}=\pi^{-1}\left(D_{\alpha}^{\mathrm{b}}\right)$, where $D_{\alpha}^{\mathrm{b}}$ are divisors in the base $\mathcal{B}_{3}$. Thus, $\bar{d}_{\alpha a b}=d_{\alpha b a}$. In terms of the real basis we have that

$$
d_{\alpha a b}=\frac{1}{2} \operatorname{Re} f_{a c}\left(M_{\alpha b}{ }^{c}+i \bar{f}^{c d} M_{\alpha d b}\right),
$$

where we defined

$$
M_{\alpha a b}=\int \omega_{\alpha} \wedge \alpha_{a} \wedge \alpha_{b}, \quad M_{\alpha a}^{b}=\int \omega_{\alpha} \wedge \alpha_{a} \wedge \beta^{b} .
$$

The action (4.6) can be expressed in terms of a Kähler potential and complex coordinates as in the weak string coupling setting. The correct complex coordinates are the $N^{a}$ as well as complex coordinates $T_{\alpha}$ containing the Kähler structure deformation defined as

$$
T_{\alpha}=\rho_{\alpha}-\frac{i}{2} d_{\alpha a b} N^{a}(N+\bar{N})^{b}-\frac{i}{2} \int_{D_{\alpha}^{\mathrm{b}}} J_{\mathrm{b}} \wedge J_{\mathrm{b}}
$$

where $J_{\mathrm{b}}$ is the Kähler form in the base. The Kähler potential is given by

$$
K=-\log \left(\int_{Y_{4}} \Omega \wedge \bar{\Omega}\right)-2 \log \mathcal{V}_{\mathrm{b}},
$$

where it is crucial to express the base volume $\mathcal{V}_{\mathrm{b}}=\frac{1}{6} \int_{\mathcal{B}_{3}} J_{\mathrm{b}} \wedge J_{\mathrm{b}} \wedge J_{\mathrm{b}}$ in terms of the complex coordinates $N^{a}, T_{\alpha}$ given in (4.10), and the complex structure deformations.

Let us now turn to the analysis of the isometries of the metric (4.6). The metric has the following holomorphic isometries

$$
\begin{aligned}
\delta N^{a} & =-i\left(\lambda^{a}+i f^{a b} \lambda_{b}\right), \\
\delta T_{\alpha} & =\lambda_{\alpha}-\frac{i}{2} N^{b} M_{\alpha a b} \lambda^{a}-N^{a}\left(i M_{\alpha a}{ }^{b}+\frac{1}{2} f^{b c} M_{\alpha c a}\right) \lambda_{b},
\end{aligned}
$$

with $\lambda^{a}, \lambda_{a}, \lambda_{\alpha}$ real. The corresponding Killing vectors read

$$
\begin{aligned}
\tilde{t}^{b} & =f^{a b} \partial_{N^{a}}-N^{a}\left(i M_{\alpha a}{ }^{b}+\frac{1}{2} f^{b c} M_{\alpha c a}\right) \partial_{T_{\alpha}}, \\
t_{a} & =-i \partial_{N^{a}}-\frac{i}{2} N^{b} M_{\alpha a b} \partial_{T_{\alpha}}, \quad t^{\alpha}=\partial_{T_{\alpha}} .
\end{aligned}
$$

It is then straightforward to check that the only non-vanishing commutator is

$$
\left[t_{a}, \tilde{t}^{b}\right]=-M_{\alpha a}{ }^{b} t^{\alpha}
$$


which again defines an algebra that is a generalisation of the Heisenberg algebra. Notice that $M_{\alpha a b}$ does not appear in the structure constants.

The expression (4.15) is the analog of the weak string coupling algebra (3.6). In fact, the setup reduces to the one of subsection 3.1 in a special limit. In order to see that one interprets all fields $N^{a}$ to arise from the bulk as the fields $G^{a}$ used in subsection 3.1. Setting

$$
f^{a b}=i \tau \delta^{a b}, \quad N^{a}=-i G^{a},
$$

one recovers the weak coupling expressions for all couplings. However, it is crucial to point out that away from weak string coupling $f^{a b}$ will in general not be diagonal. The nondiagonal generalisation will be crucial when considering the gauging of the holomorphic isometries as we discuss in the next subsection. In contrast to the weak coupling setting there can now be gauged scalars $N^{a}$ for which the real and imaginary parts are not gauged simultaneously while preserving the non-Abelian structure.

It is interesting to stress that in F-theory the $N^{a}$ also contain the Wilson line degrees of freedom. Even at weak string coupling, i.e. when considering $N^{a}$ to be Wilson line moduli for D7-branes, one finds that they couple via a holomorphic function $f^{a b}$ of the complex structure moduli and D7-brane positions. It appears that this holomorphic function does not have to be diagonal in its indices. This yields another non-trivial generalisation of the setting discussed in subsection 3.1. In F-theory the various generalisations are elegantly combined due to the combination of bulk and brane degrees of freedom in a higher-dimensional geometry.

\subsection{Non-Abelian gaugings from seven-branes - origins}

Having determined the holomorphic symmetries of the general Type IIB configuration away from the weak string coupling limit, one can now ask which subalgebra of these symmetries can be gauged. In particular, given the complications encountered for the orientifold setup in subsection 3.2, it will be crucial to argue that in more general F-theory settings a nonAbelian group can indeed be gauged. The gaugings we will discuss arise from gauge fields on general $(p, q)$-seven-branes and we also consider possible gaugings using $\mathrm{R}$-R gauge fields due to non-closed forms in the base $\mathcal{B}_{3}$. The non-closed forms can be interpreted as parameterising torsional cohomology $\operatorname{Tor} H^{3}\left(\mathcal{B}_{3}, \mathbb{Z}\right)$ similar to the discussion of subsection 3.2.

To begin with we first recall the gaugings arising when D7-branes are included in a weak string coupling scenario. If we include D7-branes wrapping a divisor $S_{i}$, the $\mathrm{U}(1)$ gauge boson $A^{i}$ with field-strength $F^{i}=d A^{i}$ may become massive due to the interaction with the closed string sector for two independent reasons, which in either case are compatible with supersymmetry.

Firstly, we consider a configuration with brane image-brane pairs in an orientifold configuration in which some of the divisors $S_{i}$ and the image-brane divisors $S_{i}^{\prime}$ are in different homology classes, i.e. situations in which some of the $S_{i}^{-}=\frac{1}{2}\left(S_{i}-S_{i}^{\prime}\right)$ are homologically non-trivial. The Chern-Simons action then contains a coupling of the form

$$
S_{\mathrm{D} 7_{i}} \supset \int_{\mathbb{R}^{1,3} \times S_{i}^{-}} C_{6} \wedge F^{i}=\tilde{k}_{i}^{a} \int_{\mathbb{R}^{1,3}} \tilde{c}_{a}^{(2)} \wedge F^{i}, \quad \tilde{k}_{i}^{a}=\int_{S_{i}^{-}} \tilde{\omega}^{a}
$$


where we have expanded $C_{6}=\tilde{c}_{a}^{(2)} \wedge \tilde{\omega}^{a}$ with $\tilde{\omega}^{a}$ being an integral harmonic four-form that is odd under the orientifold action. This induces a Stückelberg gauging of the axion $c^{a}$ dual to the two-form $\tilde{c}_{a}^{(2)}$ as

$$
D G^{a}=d G^{a}-\tilde{k}_{i}^{a} A^{i}
$$

One can show that, generically, this leads to a spontaneous breaking of the symmetry (see e.g. [30]). The surviving unbroken symmetry may contain a discrete part which is always Abelian. The details of this discrete part are discussed in [39, 45].

Secondly, there is a possibility of switching on fluxes $\mathcal{F}^{i}$ on the D7-branes. The gauging induced by this generalisation is of the form

$$
D T_{\alpha}=d T_{\alpha}-\Theta_{\alpha i} A^{i}, \quad \Theta_{\alpha i}=\int_{S_{i}} \mathcal{F}^{i} \wedge \omega_{\alpha},
$$

with $D G^{a}$ being unmodified. We note that these considerations generalize if we include several D7-branes. Taking into account the appropriate Chern-Simons couplings, we may find a discrete Abelian gauge symmetry [39, 45].

To gain a intuition how this D7-brane setting generalises, let us naively consider a configuration that contains O7-planes and $(0,1)$-seven-branes. In this case, the analogous coupling to (4.17) is

$$
S_{(0,1)} \supset \int_{\mathbb{R}^{1,3} \times S_{i}^{-}} B_{6} \wedge F^{i}=\delta^{a b} k_{b i} \int_{\mathbb{R}^{1,3}} \tilde{b}_{a}^{(2)} \wedge F^{i}
$$

with $B_{6}$ dual to the NS-NS two-form, $B_{6}=\tilde{b}_{a}^{(2)} \wedge \tilde{\omega}^{a}$. One would therefore expect that in this case the $b^{a}$ scalar, dual to $\tilde{b}_{a}^{(2)}$, receives a gauging of the form

$$
D G^{a}=d G^{a}-\tau \delta^{a b} k_{a i} A^{i} .
$$

Of course, this setting cannot be fully trusted, since we have included a $(0,1)$-seven-brane in a weak coupling scenario. We should instead return to the F-theory setting outlined in subsection 4.1 as we will do below.

Let us finally turn to the discussion of gaugings due to non-closed two-forms in the base $\mathcal{B}_{3}$. This will lead to gaugings involving the R-R gauge-fields just as in subsection 3.2. As before this requires non-closed forms to be included among the $\omega_{\alpha}$ in the base $\mathcal{B}_{3}$ such that

$$
d \omega_{\alpha}=k_{\alpha \kappa} \beta^{\kappa},
$$

where $\beta^{\kappa}$ are three-forms in $\mathcal{B}_{3}$. Carrying out the expansion of $C_{4}$ in a process similar so that shown in subsection 3.2 one finds that (4.22) induces the gauging

$$
D T_{\alpha}=d T_{\alpha}-k_{\alpha \kappa} A^{\kappa}
$$

which is of similar form as (4.19) but only uses the bulk vectors $A^{\kappa}$. The relation (4.22) can be interpreted as arising from torsional cohomology $\operatorname{Tor} H^{3}\left(\mathcal{B}_{3}, \mathbb{Z}\right) \cong \operatorname{Tor} H^{4}\left(\mathcal{B}_{3}, \mathbb{Z}\right)$ as introduced in subsection 3.2. Note that the $H^{p}\left(\mathcal{B}_{3}\right)$ have to be identified with $H_{+}^{3}\left(Y_{3}\right)$ if a double-covering Calabi-Yau threefold $Y_{3}$ exists in the weak coupling limit. We thus do not require that torsion in the negative cohomology be considered. This modification of the setting may evade the problems encountered in subsection 3.2. 


\subsection{Non-Abelian gaugings from seven-branes — gauge invariant structures}

We have just motivated that the gaugings in a Type IIB setting with $(p, q)$-seven-branes can be more general than in the weak coupling configurations of section 3.2. In order to study the system away from the weak string coupling limit we return to the configuration introduced in subsection 4.1. To gain some intuition about the gaugings that occur one can formally perform the replacement (4.16) introducing $N^{a}$ and $f^{a b}$ in the gaugings of subsection 4.2. An honest derivation, however, can only be performed via the duality with M-theory. In fact we will justify some of the following results using this duality in section 5 .

In general, one finds that only a subalgebra of the isometry algebra (4.12) discussed in subsection 4.1 will be gauged. Clearly, to define a subalgebra one has to respect various constraints ensuring, for example, the closure of this algebra. The structure constants will generically also differ from the ones of the full isometry algebra. Let us exemplify this by using a subset of the gaugings introduced in subsection 4.2. In a first F-theory example will only use seven-brane vectors in the gaugings, and hence the structure constants of the subalgebra will be of the form $\hat{f}_{i j}{ }^{k}$. Motivated by the structures which appear in (4.18), (4.19) and (4.21) we will consider a subalgebra of (4.12) that is associated with the generators

$$
X_{i}=k_{i}^{a} t_{a}-k_{i a} \tilde{t}^{a}+\Theta_{\alpha i} t^{\alpha}, \quad\left[t_{i}, t_{j}\right]=\hat{f}_{i j}{ }^{k} t_{k},
$$

which defines the structure constants $\hat{f}_{i j}{ }^{k}$. Then by using (4.15) we find that

$$
\left(\tilde{k}_{i}^{a} k_{j b}-\tilde{k}_{j}^{a} k_{i b}\right) M_{\alpha a}{ }^{b}=\hat{f}_{i j}{ }^{k} \Theta_{\alpha k}, \quad \hat{f}_{i j}{ }^{k} k_{k a}=0, \quad \hat{f}_{i j}{ }^{k} \tilde{k}_{k}^{a}=0 .
$$

We note that this analysis is not sufficient to uniquely fix the structure constants $\hat{f}_{i j}{ }^{k}$ but only certain projections on them. This is familiar from the standard embedding tensor discussions (see e.g. [46, 47]). The covariant derivatives associated with gauging this subgroup are then given by

$$
\begin{aligned}
D N^{a} & =d N^{a}+i\left(\tilde{k}_{i}^{a} A^{i}-i f^{a b} k_{i b} A^{i}\right), \\
D T_{\alpha} & =d T_{\alpha}-\Theta_{\alpha i} A^{i}+\frac{i}{2} N^{b} M_{\alpha a b} \tilde{k}_{i}^{a} A^{i}-N^{a}\left(i M_{\alpha a}{ }^{b}+\frac{1}{2} f^{b c} M_{\alpha c a}\right) k_{i b} A^{i},
\end{aligned}
$$

and the field strength $F^{i}=d A^{i}+\hat{f}_{j k}{ }^{i} A^{j} \wedge A^{k}$ is constrained such that

$$
\Theta_{\alpha i} F^{i}=\Theta_{\alpha i} d A^{i}+\tilde{k}_{k}^{a} k_{j b} M_{\alpha a}{ }^{b} A^{j} \wedge A^{k}, \quad k_{i a} F^{i}=k_{i a} d A^{i}, \quad k_{i}^{a} F^{i}=k_{i}^{a} d A^{i} .
$$

If we recall that, roughly speaking, $\tilde{k}_{j}^{a}$ labels $(1,0)$-brane part of the gauging, $k_{j b}$ is the $(0,1)$-brane part of the gauging, and $M_{\alpha a}{ }^{b}$ is the non-trivial twisting of the moduli space metric (4.6), then we see that it is the presence of gaugings associated with mutually nonlocal seven-branes that is crucial for generating the non-Abelian structure in (4.27). In addition to this we see that the non-Abelian structure is linked to the presence of fluxes in this picture. It is well-known that fluxes induce chirality and accordingly the classical action does not need to be gauge invariant as discussed briefly at the end of subsection 2.2. 
The second example of non-Abelian gaugings occurring in F-theory is obtained by switching off fluxes on the seven-branes (i.e. setting $\Theta_{\alpha i}=0$ ) and turning on $k_{\alpha \kappa}$ appearing in (4.22). It will be this example that we will study in much more detail using the Mtheory dual in section 5. Analysing the subalgebra spanned by $\tilde{k}_{j}^{a}, k_{i a}$, and $k_{\alpha \kappa}$, we find that the only non-vanishing structure constants are in this case of the form $\hat{f}_{i j}{ }^{\kappa}$. They are constrained only by

$$
\hat{f}_{i j}{ }^{\kappa} \Pi_{\kappa}{ }^{\lambda}=\left(\tilde{k}_{i}^{a} k_{j b}-\tilde{k}_{j}^{a} k_{i b}\right) M_{\alpha a}{ }^{b} \check{k}^{\lambda \alpha},
$$

and thus for the gauged subalgebra to close we must demand that

$$
\Pi_{\alpha}^{\beta}\left(\tilde{k}_{j}^{a} k_{i b}-\tilde{k}_{i}^{a} k_{j b}\right) M_{\beta a}{ }^{b}=\left(\tilde{k}_{j}^{a} k_{i b}-\tilde{k}_{i}^{a} k_{j b}\right) M_{\alpha a}{ }^{b} .
$$

In these equations we have defined the projectors $\Pi_{\kappa}{ }^{\lambda}$ and $\Pi_{\alpha}{ }^{\beta}$ as well as the Moore-Penrose pseudo-inverse $\check{k}^{\kappa \alpha}$ of $k_{\alpha \kappa}$. These quantities satisfy

$$
k_{\alpha \kappa} \check{k}^{\lambda \alpha}=\Pi_{\kappa}^{\lambda}, \quad k_{\alpha \kappa} \check{k}^{\kappa \beta}=\Pi_{\alpha}^{\beta}, \quad \Pi_{\kappa}{ }^{\lambda} k_{\alpha \lambda}=k_{\alpha \kappa}, \quad \Pi_{\alpha}^{\beta} k_{\beta \kappa}=k_{\alpha \kappa} .
$$

In this case the gaugings (4.26) are replaced by

$$
\begin{aligned}
D N^{a} & =d N^{a}+i\left(\tilde{k}_{i}^{a} A^{i}-i f^{a b} k_{i b} A^{i}\right), \\
D T_{\alpha} & =d T_{\alpha}-k_{\alpha \kappa} A^{\kappa}+\frac{i}{2} N^{b} M_{\alpha a b} \tilde{k}_{i}^{a} A^{i}-N^{a}\left(i M_{\alpha a}{ }^{b}+\frac{1}{2} f^{b c} M_{\alpha c a}\right) k_{b i} A^{i},
\end{aligned}
$$

and the field strengths are constrained such that

$$
k_{\alpha \kappa} F^{\kappa}=k_{\alpha \kappa} d A^{\kappa}+\tilde{k}_{j}^{a} k_{k b} M_{\alpha a}^{b} A^{j} \wedge A^{k}, \quad \quad F^{i}=d A^{i} .
$$

We stress that in this case only the R-R bulk gauge-field admits a non-Abelian modification.

This second possibility of obtaining non-Abelian gaugings has the advantage of being purely geometrically induced. In particular, one expects following $[6,31-33]$ that the geometrically massive gauge fields gauging $N^{a}$ are obtained from non-closed forms on the Calabi-Yau fourfold in M-theory. Together with the possibly non-closed two-forms $\omega_{\alpha}$ satisfying (4.22), one thus expects to find a geometric M-theory reduction that yields precisely the gaugings (4.31) upon lifting to F-theory. We will show in section 5 that this is indeed the case. Furthermore, we are able to directly determine the structure constants $\hat{f}_{i j}{ }^{\kappa}$ to be given by

$$
\hat{f}_{i j}{ }^{\kappa}=\tilde{k}_{[j}^{a} M_{i] a}{ }^{\kappa}+k_{[j a} M_{i]}^{a \kappa} \text {. }
$$

Here $M_{i a}{ }^{\kappa}$ and $M_{i}{ }^{a \kappa}$ are constant coupling matrices that are explicitly given in section 5 .

To get an idea about the meaning of these couplings, let us give their weak string coupling expressions in the Calabi-Yau orientifold setting $\mathcal{B}_{3}=Y_{3} / \sigma$. If the index $a$ counts bulk scalars $G^{a}$ then we find for D7-branes

$$
M_{i a}{ }^{\kappa}=0, \quad M_{i}^{a \kappa}=\delta^{a b} \int_{\mathcal{C}^{i}} \omega_{b} \wedge \beta^{\kappa},
$$

where $\mathcal{C}^{i}$ is a chain ending on the $i$ th D7-brane world-volume and $\omega_{a}$ is the orientifold-odd harmonic two-form on $Y_{3}$. Note that $M_{i a}{ }^{\kappa}$, as defined in section 5 , should only include the 
constant part of the chain integral in (4.34). Once again we can see that the non-Abelian gaugings disappear for settings with only D7-branes, since in this case $k_{i a}=0$ and $M_{i a}{ }^{k}=0$ in (4.33).

Alternatively the index $a$ could also parameterise Wilson line moduli on the sevenbranes. Let us introduce one-forms $\left(\gamma_{a_{i}}, \gamma^{b_{i}}\right)$ on the $i$ th seven-brane with world-volume $S^{i}$. Then we find that

$$
M_{i a_{i}}{ }^{\kappa}=\int_{S^{i}} \gamma_{a_{i}} \wedge \beta^{\kappa}, \quad M_{i}^{a_{i} \kappa}=\int_{S^{i}} \gamma^{a_{i}} \wedge \beta^{\kappa}
$$

In this case one finds indeed that both $M_{i a_{i}}{ }^{\kappa}$ and $M_{i}{ }^{a_{i} \kappa}$ are non-zero. However, in order to realise a non-Abelian symmetry with non-vanishing (4.33) we need to gauge the Wilson line scalars. We are not aware that such a setting has been investigated yet.

Let us close this section with another crucial observation which ties in with the discussion of the gauge coupling function presented at the end of subsection 2.2. It also explains how we were able to deduce the expressions (4.34) and (4.35). It turns out, as we will see in section 5 , that the $M_{i a}{ }^{\kappa}$ and $M_{i}{ }^{a \kappa}$ precisely encode the kinetic mixing of the R-R gauge fields $A^{\kappa}$ and the seven-brane gauge-fields. More precisely, we find

$$
\begin{aligned}
\operatorname{Re} f_{\lambda i} & =\operatorname{Re} f_{\lambda \kappa}\left(M_{i a}{ }^{\kappa} a^{a}-M_{i}{ }^{a \kappa} b_{a}\right), \\
\operatorname{Re} f_{i j} & =\check{G}_{i j}+\operatorname{Re} f_{\lambda \kappa}\left(M_{i a}{ }^{\kappa} a^{a}-M_{i}{ }^{a \kappa} b_{a}\right)\left(M_{j a}{ }^{\lambda} a^{a}-M_{j}{ }^{a \lambda} b_{a}\right),
\end{aligned}
$$

where $f_{\lambda \kappa}$ is the holomorphic gauge coupling function of the R-R gauge fields $A^{\kappa}$, and $\check{G}_{i j}$ is a Kähler moduli dependent metric. The fact that the gauge couplings depend on the scalars $\left(a^{a}, b_{a}\right)$ nicely matches the requirement that for a gauged non-semisimple and non-compact group this coupling needs to transform non-trivially. The kinetic mixing (4.36) is present independent of the gaugings, i.e. even if we set $\tilde{k}_{a}^{i}=k_{i a}=0$ and $k_{\alpha \kappa}=0$. If we allow for non-Abelian gaugings then the terms in (4.36) are actually essential for gauge-invariance. Let us note that the results (4.34) and (4.35) were deduced by comparing the kinetic mixing terms on seven-branes with (4.36). Kinetic mixing on D7-branes was studied also in $[34,48,49]$. One therefore expects that (4.36) can be made the real part of a holomorphic function in the correct $\mathcal{N}=1$ complex coordinates as required by supersymmetry. We leave the details of this investigation to a further publication [50].

Let us close this section by stressing some of the differences to the discrete Abelian symmetries recently considered in [17,22-28]. As of now, most of the considerations were for the effective theory and the continuous non-Abelian symmetry. Focusing on the vacua of the theory one expects that there is, in contrast to the Abelian case, no vacuum in which a continuous non-Abelian group is preserved. This can be inferred from the fact that the background gauge coupling function can not be positive definite and invariant as no such tensor exists. In the Abelian case a more complete analysis was possible and it was argued that in this case there exists a transition in complex structure moduli space that restores a global U(1) symmetry. 


\section{Non-Abelian discrete symmetries via F-/M-theory duality}

In this section we use the duality of M-theory and F-theory to show the appearance of discrete non-Abelian gauge symmetries in F-theory as claimed in section 4. More precisely, we perform a dimensional reduction of eleven-dimensional supergravity including a number of non-harmonic forms. These forms might be viewed as representing torsion cohomology elements. ${ }^{4}$ The three-dimensional effective action is determined in subsection 5.1 and possesses only Abelian gaugings. The non-Abelian gaugings arise when bringing the three-dimensional action into the duality frame relevant for the F-theory up-lift to four dimensions. The relevant dualisations of the fields are discussed in subsection 5.2 and appendix A. We are then able to show that the covariant derivatives (4.31) and field strengths (4.32) are reproduced by the reduction. We also find that the structure constants are given by (4.33) and the gauge coupling function takes the form (4.36).

\subsection{Non-harmonic reduction of M-theory}

Recall that the duality between M-theory and F-theory asserts that compactifying the former theory on an elliptically fibered Calabi-Yau manifold is dual to the latter theory on the same manifold times a circle. The comparison of effective theories of M-theory and F-theory is therefore performed in three dimensions. One can thus start with a candidate four-dimensional action, the F-theory effective action, and compactify the theory on a circle. The lower-dimensional theory can be pushed to the Coulomb branch and all heavy modes, including the Kaluza-Klein states, can be integrated out to obtain the effective theory for massless states only. However, we claim that the M-theory reduction will also contain massive modes that arise due to the inclusion of non-harmonic forms. Therefore, we have to carefully keep track of certain charged or massive states in the matching of the M-theory and F-theory actions. This is in complete analogy to the case in which one considers background fluxes. In the following we will thus discuss three-dimensional gauged supergravity theories to justify the F-theory effective action of section 4 . Our main focus will be on inferring the couplings (4.31), (4.27), and (4.36), which dictate the presence of a non-Abelian gauge symmetry.

The M-theory reduction is performed by using eleven-dimensional supergravity. This implies that we have to work with a resolved fourfold $\hat{Y}_{4}$. Furthermore, all linearly charged matter states corresponding to M2-branes on the resolution cycles are integrated out and will not appear in the following three-dimensional effective action. The starting action is the bosonic part of eleven-dimensional supergravity given by

$$
S^{(11)}=\frac{1}{2} \int\left(\hat{R} \hat{*} 1-\frac{1}{2} \hat{G} \wedge \hat{*} \hat{G}-\frac{1}{6} \hat{C} \wedge \hat{G} \wedge \hat{G}\right)
$$

where $\hat{R}$ is the eleven-dimensional Ricci scalar and $\hat{G}=d \hat{C}$ is the four-form field strength for the three-form $\hat{C}$. In the following a hat will indicate that the quantity is defined in eleven dimensions.

\footnotetext{
${ }^{4}$ While we believe that Calabi-Yau four-folds with such torsion cohomology exist, we refrain from constructing explicit examples.
} 
Clearly, the M-theory reduction should not only include harmonic forms, but also contain non-closed and exact forms that account for possible gaugings. These forms can be viewed as parameterising torsion cohomology. We thus introduce the two-forms $\omega_{\Sigma}$, and three-forms $\left(\alpha_{I}, \beta^{I}\right)$ on $\hat{Y}_{4}$ that need not be harmonic but should be definite eigenstates of the Laplace-Beltrami operator. They are related by the non-closure of $\omega_{\Sigma}$ given by

$$
d \omega_{\Sigma}=\tilde{k}_{\Sigma}^{I} \alpha_{I}+k_{\Sigma I} \beta^{I} .
$$

This expression is a generalisation of (4.22) in which $\omega_{\alpha}$ and $\beta^{\kappa}$ are elements of the base $\mathcal{B}_{3}$ of $\hat{Y}_{4}$. It also contains the case that $\omega_{\Sigma}$ yields a gauge field of a seven-brane and the nonclosure yields the gaugings induced from the geometric Stückelberg term (4.17) and (4.20). For D7-branes this has already been suggested in [6, 31, 33].

Next we introduce the modes of the effective theory that arise from expanding the eleven-dimensional metric and the M-theory three-form into $\omega_{\Sigma}$ and $\left(\alpha_{I}, \beta^{I}\right)$. We will therefore make an ansatz for the reduction where

$$
\begin{aligned}
d \hat{s}^{2} & =g_{\mu \nu} d x^{\mu} d x^{\nu}+2\left(g_{m \bar{n}}^{0}+i \delta v^{\Sigma} \omega_{\Sigma m \bar{n}}\right) d y^{m} d y^{\bar{n}}, \\
\hat{C} & =A^{\Sigma} \wedge \omega_{\Sigma}+\tilde{\xi}^{I} \alpha_{I}+\xi_{I} \beta^{I} .
\end{aligned}
$$

In this expression $\delta v^{\Sigma}, \tilde{\xi}^{I}$ and $\xi_{I}$ are three-dimensional scalar fields, while $A^{\Sigma}$ are threedimensional vector fields. Note that $\delta v^{\Sigma}$ parameterise the deformations of the Calabi-Yau metric $g_{m \bar{n}}^{0}$ that are in general non-Kähler. Setting $J=J_{0}+\delta v^{\Sigma} \omega_{\Sigma}$ one has

$$
d J=\delta v^{\Sigma} d \omega_{\Sigma}=\delta v^{\Sigma} \tilde{k}_{\Sigma}^{I} \alpha_{I}+\delta v^{\Sigma^{2}} k_{\Sigma I} \beta^{I},
$$

which implies that there will be a potential induced for the scalars $\delta v^{\Sigma}$. We will denote the complete three-dimensional scalar potential by $V$, but will refrain discussing its precise form. We will also introduce the scalars $v^{\Sigma}$, which parameterise the expansion of $J=v^{\Sigma} \omega_{\Sigma}$. More important in the following is the reduction of the M-theory three-form part of the action. Using (5.4) and (5.2) we see that $\hat{G}$ is given by

$$
\hat{G}=d A^{\Sigma} \wedge \omega_{\Sigma}+D \tilde{\xi}^{I} \wedge \alpha_{I}+D \xi_{I} \wedge \beta^{I}+\tilde{\xi}^{I} d \alpha_{I}+\xi_{I} d \beta^{I}
$$

Here we have defined the covariant derivatives

$$
D \tilde{\xi}^{I}=d \tilde{\xi}^{I}-A^{\Sigma} \tilde{k}_{\Sigma}^{I}, \quad D \xi_{I}=d \xi_{I}-A^{\Sigma} k_{\Sigma I} .
$$

As we will show in the following it will be these simple gaugings that are responsible for the gauge structure encountered in the F-theory effective action of section 4.

Substituting the ansatz (5.4) and (5.5) into the action (5.1) and performing a Weyl rescaling, which puts the effective action in Einstein frame, we find the three-dimensional effective theory given by

$$
\begin{aligned}
S^{(3)}= & \frac{1}{2} \int\left[R * 1-\frac{1}{2} G_{\Sigma \Lambda} d L^{\Sigma} \wedge * d L^{\Lambda}-\frac{1}{2} G_{\Sigma \Lambda} F^{\Sigma} \wedge * F^{\Lambda}\right. \\
& -\frac{1}{2} \tilde{G}_{I J} D \tilde{\xi}^{I} \wedge * D \tilde{\xi}^{J}-\frac{1}{2} G^{I J} D \xi_{I} \wedge * D \xi_{J}-H_{I}^{J} D \tilde{\xi}^{I} \wedge * D \xi_{J}
\end{aligned}
$$




$$
\begin{aligned}
& +\frac{1}{3} M_{\Sigma I} F^{\Sigma} \wedge\left(\tilde{\xi}^{I} D \xi_{J}-\xi_{J} D \tilde{\xi}^{I}\right)+\frac{1}{3} M_{\Sigma I J} F^{\Sigma} \wedge \tilde{\xi}^{I} D \tilde{\xi}^{J}+\frac{1}{3} M_{\Sigma}^{I J} F^{\Sigma} \wedge \xi_{I} D \xi_{J} \\
& +\frac{1}{3} M_{\Sigma I} A^{\Sigma} \wedge D \tilde{\xi}^{I} \wedge D \xi_{J}+\frac{1}{6} M_{\Sigma I J} A^{\Sigma} \wedge D \tilde{\xi}^{I} \wedge D \tilde{\xi}^{J}+\frac{1}{6} M_{\Sigma}^{I J} A^{\Sigma} \wedge D \xi_{I} \wedge D \xi_{J} \\
& \left.-\frac{1}{3} N_{\Sigma \Lambda I} \tilde{\xi}^{I} A^{\Sigma} \wedge F^{\Lambda}+\frac{1}{3} \tilde{N}_{\Sigma \Lambda}{ }^{I} \xi_{I} A^{\Sigma} \wedge F^{\Lambda}+V * 1\right]
\end{aligned}
$$

The first line contains the kinetic terms for the scalars $v^{\Sigma}$ and vectors $A^{\Sigma}$. To write them in this simple form, we have used the definitions

$$
G_{\Sigma \Lambda}=\mathcal{V} \int_{\hat{Y}_{4}} \omega_{\Sigma} \wedge * \omega_{\Lambda}, \quad \quad L^{\Sigma}=\frac{v^{\Sigma}}{\mathcal{V}},
$$

where $\mathcal{V}$ is the volume of the manifold $\hat{Y}_{4}$. To display the couplings of the scalars $\left(\xi_{I}, \tilde{\xi}^{J}\right)$ we have introduced the definitions

$$
\begin{aligned}
\tilde{G}_{I J} & =\frac{1}{\mathcal{V}} \int_{\hat{Y}_{4}} \alpha_{I} \wedge * \alpha_{J}, & G^{I J} & =\frac{1}{\mathcal{V}} \int_{\hat{Y}_{4}} \beta^{I} \wedge * \beta^{J}, \\
H_{I}{ }^{J} & =\frac{1}{\mathcal{V}} \int_{\hat{Y}_{4}} \alpha_{I} \wedge * \beta^{J}, & M_{\Sigma I}^{J} & =\int_{\hat{Y}_{4}} \omega_{\Sigma} \wedge \alpha_{I} \wedge \beta^{J}, \\
N_{\Sigma \Lambda I} & =\int_{\hat{Y}_{4}} \omega_{\Sigma} \wedge \omega_{\Lambda} \wedge d \alpha_{I}, & \tilde{N}_{\Sigma \Lambda}^{I} & =-\int_{\hat{Y}_{4}} \omega_{\Sigma} \wedge \omega_{\Lambda} \wedge d \beta^{I} .
\end{aligned}
$$

The tensors $N_{\Sigma \Lambda I}$ and $\tilde{N}_{\Sigma \Lambda}^{I}$ can be written in terms of the other couplings by integrating by parts and using (5.2), which gives

$$
\begin{aligned}
N_{\Sigma \Lambda I} & =k_{\Sigma J} M_{\Lambda I}^{J}+\tilde{k}_{\Sigma}^{J} M_{\Lambda I J}+k_{\Lambda J} M_{\Sigma I}^{J}+\tilde{k}_{\Lambda}^{J} M_{\Sigma I J}, \\
\tilde{N}_{\Sigma \Lambda}^{I} & =k_{\Sigma J} M_{\Lambda}^{J I}+\tilde{k}_{\Sigma}^{J} M_{\Lambda J}{ }^{I}+k_{\Lambda J} M_{\Sigma}^{J I}+\tilde{k}_{\Lambda}^{J} M_{\Sigma J}^{I}, \\
k_{\Lambda I} N_{\Sigma \Delta}^{I} & =\tilde{k}_{\Lambda}^{I} N_{\Sigma \Delta I} .
\end{aligned}
$$

Let us close this subsection with a few crucial observations. It is straightforward to see that the action (5.7) enjoys an Abelian gauge symmetry given by

$$
\delta A^{\Sigma}=d \lambda^{\Sigma}, \quad \delta \tilde{\xi}^{I}=\tilde{k}_{\Sigma}^{I} \lambda^{\Sigma}, \quad \delta \xi_{I}=k_{\Sigma I} \lambda^{\Sigma},
$$

where $\lambda^{\Sigma}$ is a gauge parameter. However, in the last sections we argued that this system should posses non-Abelian symmetries. Surprisingly, such a non-Abelian structure is present in this setup although it is not obviously realised in terms of the fields we introduced. In the next section we will see how by performing a change of duality frame for certain fields we unravel the non-Abelian symmetries. This new frame turns out to be the correct one in which to perform the F-theory limit and so compare with the fourdimensional effective theory.

A final comment concerns the supersymmetry properties of the action (5.7). We have not demonstrated that this action is indeed supersymmetric. In order to do that one would have to introduce complex coordinates on the moduli space and demonstrate that the couplings in (5.7) are of special form, e.g. obtained from a Kähler potential. This requires the introduction of $(2,1)$-forms on $\hat{Y}_{4}$ that can be parameterised by a holomorphic 
function varying over the complex structure moduli space. This function is then used in defining the complex coordinates in generalisation of (4.3) and (4.5). While the ungauged action can then be shown to be supersymmetric, it is expected that additional conditions on the allowed gaugings are imposed by supersymmetry. It would be nice to determine these conditions from a more detailed analysis of the geometry. In the following we will continue with our analysis on the bosonic action and focus on the appearance of the non-Abelian gaugings manifested through (4.31) and (4.32).

\subsection{Dualisation of the M-theory effective action}

The action (5.7), obtained by dimensional reduction of eleven-dimensional supergravity, is not yet in the duality frame that allows a lift to a four-dimensional F-theory configuration. In the following we will perform a dualisation to bring it into the correct form. In order to do this we must first split the three-dimensional fields into those which are affected by the duality and those which are not. For this reason we will make the decomposition

$$
L^{\Sigma}=\left(L^{\hat{\imath}}, L^{\alpha}\right), \quad A^{\Sigma}=\left(A^{\hat{\imath}}, A^{\alpha}\right), \quad \tilde{\xi}^{I}=\left(a^{a}, \tilde{\xi}^{\kappa}\right), \quad \xi_{I}=\left(-b_{a}, \xi_{\kappa}\right) .
$$

This is in complete analogy to the ungauged case [44]. The multiplet $\left(L^{\alpha}, A^{\alpha}\right)$ will lift to the bosonic part of a four-dimensional chiral multiplet with scalars $T_{\alpha}$ and therefore $A^{\alpha}$ needs to be dualised into a scalar $\rho_{\alpha}$ in three dimensions. In contrast $\left(\tilde{\xi}^{\kappa}, \xi_{\kappa}\right)$ will comprise the degrees of freedom of a vector in a four-dimensional vector multiplet. These are the four-dimensional R-R vectors $A^{\kappa}$ used in (4.31). Therefore one must dualise the scalar $\xi_{\kappa}$ into a three-dimensional vector $A^{\kappa}$ before performing the uplift. Note that in this section we slightly abuse notation and assert that $A^{\kappa}$ and $A^{\hat{\imath}}$ are three-dimensional vectors. Finally the multiplet $\left(a^{a}, b_{a}\right)$ lifts to chiral multiplet in the four-dimensional theory which originates from either Type IIB bulk fields, decomposed with respect to internal space two-forms, or from Wilson lines.

In order to make contact with section 4 and to keep the discussion simple, we restrict to the case in which

$$
d \omega_{\alpha}=k_{\alpha \kappa} \beta^{\kappa}, \quad d \omega_{\hat{\imath}}=\tilde{k}_{\hat{\imath}}^{a} \alpha_{a}+k_{\hat{\imath} a} \beta^{a} .
$$

The first condition is the non-closure of forms $\omega_{\alpha}$ stemming from the base $\mathcal{B}_{3}$ and agrees with (4.22). The second condition accounts for the geometric Stückelberg gaugings with the seven-brane gauge fields. It is important to stress that the dualisation we are preforming only works if we impose additional conditions relating the constant couplings and gaugings. Concretely, we find that the duality can be performed when imposing

$$
\begin{array}{rlrl}
\tilde{k}_{\hat{\imath}}^{a} M_{\hat{\jmath} a}{ }^{\kappa}+k_{\hat{\jmath} a} M_{\hat{\imath}}{ }^{a \kappa} & =0, & \\
k_{\hat{\imath} b} M_{\alpha a}{ }^{b}+k_{\alpha \kappa} M_{\hat{\imath} a}{ }^{\kappa} & =0, & & \tilde{k}_{\hat{\imath}}^{b} M_{\alpha a b}=0, \\
\tilde{k}_{\hat{\imath}}^{b} M_{\alpha b}{ }^{a}+k_{\alpha \kappa} M_{\hat{\imath}}{ }^{\kappa a} & =0, & k_{\hat{\imath} b} M_{\alpha}{ }^{b a}=0 .
\end{array}
$$

It is not clear whether these are the weakest conditions that have to be imposed. In particular, it appears that imposing only the sum of the expressions in the last two lines, 
yielding $N_{\hat{\imath} \alpha a}=\tilde{N}_{\hat{\imath} \alpha}^{a}=0$ by using (5.10) and (5.13), is also sufficient. It would be interesting to give a precise geometric reasoning why in an elliptically fibered geometry these vanishing conditions are imposed. It appears that these conditions are crucial to perform the F-theory up-lift. This can be compared with the vanishing conditions of $[13,51,52]$ on the $G_{4}$-flux intersections $\Theta_{\Lambda \Sigma}=\int \omega_{\Lambda} \wedge \omega_{\Sigma} \wedge G_{4}$ that need to be imposed for a four-dimensional gaugeinvariant setting to exist.

In addition there is a set of constraints that is readily inferred for an elliptically fibered space by counting the number of legs in the fiber. These are given by

$$
M_{\alpha \kappa}{ }^{\lambda}=M_{\alpha \kappa}{ }^{a}=M_{\alpha a}{ }^{\kappa}=M_{\alpha}{ }^{\kappa \lambda}=M_{\alpha}{ }^{\kappa a}=M_{\alpha \kappa \lambda}=M_{\alpha \kappa a}=M_{\hat{\imath}}{ }^{\kappa \lambda}=M_{\hat{\imath} \kappa \lambda}=0,
$$

which we will see is true for the duality splitting assignments appropriate for the F-theory lift.

In order to perform the duality, we proceed in the usual way. We propose a parent Lagrangian that is a function of both the original and dual fields such that it gives back the starting action (5.7) when we remove the dual fields by using their equations of motion. Alternatively, we can use the equations of motion for the original fields to remove these in favour of the dual ones which gives the dual action. This is a rather complicated computation so we simply quote the result here and refer the reader to appendix A for the details. A more detailed analysis of this Abelian to non-Abelian duality in various dimension will appear in an upcoming paper [53]. The dual Lagrangian is given by

$$
\begin{aligned}
S= & \frac{1}{2} \int\left[R * 1-\frac{1}{2} G_{\hat{\imath} \jmath} d L^{\hat{\imath}} \wedge * d L^{\hat{\jmath}}-\frac{1}{2} G_{\alpha \beta} d L^{\alpha} \wedge * d L^{\beta}-G_{\hat{\imath} \alpha} d L^{\hat{\imath}} \wedge * d L^{\alpha}\right. \\
& -\frac{1}{2} \tilde{G}_{a b} D a^{a} \wedge * D a^{b}-\frac{1}{2} \tilde{G}^{a b} D b_{a} \wedge * D b_{b}+\tilde{H}_{a}{ }^{b} D a^{a} \wedge * D b_{b}-\frac{1}{2} G^{-1 \alpha \beta} \hat{D} \rho_{\alpha} \wedge * \hat{D} \rho_{\beta} \\
& -\frac{1}{2} \tilde{G}_{\kappa \lambda} D \tilde{\xi}^{\kappa} \wedge * D \tilde{\xi}^{\lambda}-\tilde{G}_{a \kappa} D a^{a} \wedge * D \tilde{\xi}^{\kappa}+\tilde{H}_{\kappa}{ }^{a} D b_{a} \wedge * D \tilde{\xi}^{\kappa}-\frac{1}{2} G^{-1}{ }_{\kappa \lambda} U^{\kappa} \wedge * U^{\lambda} \\
& -\frac{1}{2} \tilde{G}_{\hat{\imath} \hat{\jmath}} F^{\hat{\imath}} \wedge * F^{\hat{\jmath}}-\frac{1}{3} M_{\hat{\imath} a b} a^{a} D a^{b} \wedge F^{\hat{\imath}}-G^{-1}{ }_{\kappa \lambda} G^{a \kappa} D b_{a} \wedge U^{\lambda}+G^{-1}{ }_{\kappa \lambda} H_{a}{ }^{\kappa} D a^{a} \wedge U^{\lambda} \\
& +G^{-1}{ }_{\lambda \eta} H_{\kappa}{ }^{\lambda} D \tilde{\xi}^{\kappa} \wedge U^{\eta}-G^{-1 \alpha \beta} G_{\beta \hat{\imath}} \hat{D} \rho_{\alpha} \wedge F^{\hat{\imath}}+\frac{1}{3} M_{\hat{\imath} \kappa}{ }^{a} \tilde{\xi}^{\kappa} D b_{a} \wedge F^{\hat{\imath}} \\
& +\frac{1}{3} M_{\hat{\imath} a}{ }^{b} a^{a} D b_{b} \wedge F^{\hat{\imath}}-\frac{1}{3} M_{\hat{\imath} a \kappa} a^{a} D \tilde{\xi}^{\kappa} \wedge F^{\hat{\imath}}-\frac{1}{3} M_{\hat{\imath} b}{ }^{a} b_{a} D a^{b} \wedge F^{\hat{\imath}} \\
& -\frac{1}{3} M_{\hat{\imath}}{ }^{a b} b_{a} D b_{b} \wedge F^{\hat{\imath}}-\frac{1}{3} M_{\hat{\imath} \kappa}{ }^{a} b_{a} D \tilde{\xi}^{\kappa} \wedge F^{\hat{\imath}}+\frac{1}{3} M_{\hat{\imath} a \kappa} \tilde{\xi}^{\kappa} D a^{a} \wedge F^{\hat{\imath}} \\
& -\frac{1}{3} M_{\hat{\imath} a \kappa} A^{\hat{\imath}} \wedge D a^{a} \wedge D \tilde{\xi}^{\kappa}-\frac{1}{3} M_{\hat{\imath} \kappa}{ }^{a} A^{\hat{\imath}} \wedge D b_{a} \wedge D \tilde{\xi}^{\kappa}+\frac{1}{3} M_{\hat{\imath} a}{ }^{b} A^{\hat{\imath}} \wedge D a^{a} \wedge D b_{b} \\
& \left.-\frac{1}{6} M_{\hat{\imath}}^{a b} A^{\hat{\imath}} \wedge D b_{a} \wedge D b_{b}-\frac{1}{6} M_{\hat{\imath} a b} A^{\hat{\imath}} \wedge D a^{a} \wedge D a^{b}+V * 1\right]
\end{aligned}
$$

where, as promised, we traded the scalars $\xi_{\kappa}$ and vectors $A^{\alpha}$, for the vectors $A^{\kappa}$ and scalars $\rho_{\alpha}$ respectively. It should be stressed that for simplicity we did not take into account the moduli dependence of the coupling functions. In particular, we have frozen the complex structure moduli in all these considerations. The coupling functions appearing in the 
dualised action (5.16) are defined as

$$
\begin{array}{rlrl}
\tilde{H}_{a}{ }^{b} & =H_{a}{ }^{b}-G^{b \lambda} G^{-1}{ }_{\kappa \lambda} H_{a}{ }^{\kappa}, & \tilde{G}_{a \kappa} & =\tilde{G}_{a \kappa}-G^{-1}{ }_{\lambda \eta} H_{a}{ }^{\lambda} H_{\kappa}{ }^{\eta}, \\
\tilde{H}_{\kappa}{ }^{a} & =H_{\kappa}{ }^{a}-G^{a \eta} G^{-1}{ }_{\lambda \eta} H_{\kappa}{ }^{\lambda}, & \tilde{G}_{a b} & =\tilde{G}_{a b}-G^{-1}{ }_{\kappa \lambda} H_{a}{ }^{\kappa} H_{b}{ }^{\lambda}, \\
\tilde{G}^{a b} & =G^{a b}-G^{a \kappa} G^{b \lambda} G^{-1}{ }_{\kappa \lambda}, & & \tilde{G}_{\kappa \lambda}=\tilde{G}_{\kappa \lambda}-G^{-1}{ }_{\eta \rho} H_{\kappa}{ }^{\eta} H_{\lambda}{ }^{\rho}, \\
\tilde{G}_{\hat{\imath} \hat{\jmath}}=G_{\hat{\imath} \hat{\jmath}}-G^{-1 \alpha \beta} G_{\alpha \hat{\imath}} G_{\beta \hat{\jmath}} . & &
\end{array}
$$

The action includes the gauge-invariant expressions

$$
\begin{aligned}
\hat{D} \rho_{\alpha} & =D \rho_{\alpha}+\frac{1}{2} M_{\alpha a}{ }^{b}\left(a^{a} D b_{b}-b_{b} D a^{a}\right)-\frac{1}{2} M_{\alpha a b} a^{a} D a^{b}-\frac{1}{2} M_{\alpha}{ }^{a b} b_{a} D b_{b}, \\
U^{\kappa} & =F^{\kappa}+M_{\hat{\imath} \lambda}{ }^{\kappa} \tilde{\xi}^{\lambda} F^{\hat{\imath}}+M_{\hat{\imath} a}{ }^{\kappa} a^{a} F^{\hat{\imath}}-M_{\hat{\imath}}{ }^{\kappa} b_{a} F^{\hat{\imath}},
\end{aligned}
$$

where

$$
\begin{aligned}
D \rho_{\alpha} & =d \rho_{\alpha}-k_{\alpha \kappa} A^{\kappa}-\frac{1}{2} k_{\alpha \kappa} M_{\hat{\imath} a}{ }^{\kappa} a^{a} A^{\hat{\imath}}+\frac{1}{2} k_{\alpha \kappa} M_{\hat{\imath}}{ }^{a \kappa} b_{a} A^{\hat{\imath}}, \\
F^{\kappa} & =d A^{\kappa}+\frac{1}{2}\left(\tilde{k}_{\hat{\jmath}}^{a} M_{\hat{\imath} a}{ }^{\kappa}+k_{\hat{\jmath} a} M_{\hat{\imath}}{ }^{a \kappa}\right) A^{\hat{\imath}} \wedge A^{\hat{\jmath}} .
\end{aligned}
$$

We expect that the non-Abelian structure is linked to the coupling $M_{\alpha a}{ }^{b}$. This is not obvious from the expressions above but we may use the relations (5.14) to make it manifest, namely

$$
\begin{aligned}
D \rho_{\alpha} & =d \rho_{\alpha}-k_{\alpha \kappa} A^{\kappa}+\frac{1}{2} M_{\alpha a}^{b}\left(k_{\hat{\imath} b} a^{a}-\tilde{k}_{\hat{\imath}}^{a} b_{b}\right) A^{\hat{\imath}}, \\
k_{\alpha \kappa} F^{\kappa} & =k_{\alpha \kappa} d A^{\kappa}+\tilde{k}_{\hat{\jmath}}^{a} k_{\hat{\imath} b} M_{\alpha a}{ }^{b} A^{\hat{\imath}} \wedge A^{\hat{\jmath}} .
\end{aligned}
$$

This also shows that the field strength satisfies the projection condition (4.32) that was required for closure of the gauged subalgebra.

Let us now split the index $\hat{\imath}$ further into $(0, i)$. This allows us to denote $\omega_{0}$ as the twoform Poincaré dual to the base of the elliptic fibration (which we assume to be closed in the following), $\omega_{i}$ as dual to blow-up divisors and $\omega_{\alpha}$ as dual to vertical divisors. Similarly we now understand the splitting $\alpha_{I}=\left(\alpha_{a}, \alpha_{\kappa}\right)$ and $\beta^{I}=\left(\beta^{a}, \beta^{\kappa}\right)$ as being such that $\alpha^{a}$ and $\beta_{a}$ have a component with one leg in the fiber while $\alpha_{\kappa}$ and $\beta^{\kappa}$ have legs only in the base directions. This decomposition justifies the constraints (5.15) which may be seen by counting legs of the forms present. We also now impose that

$$
M_{0 \kappa}{ }^{\lambda}=\delta_{\kappa}^{\lambda}, \quad M_{i \kappa}{ }^{\lambda}=0,
$$

the first of which shows that $\alpha_{\kappa}$ and $\beta^{\kappa}$ form a symplectic basis for three-forms on the base. With this decomposition we see that the gaugings decompose as $\tilde{k}_{\hat{\imath}}^{a}=\left(0, \tilde{k}_{i}^{a}\right)$ and $k_{\hat{\imath} a}=\left(0, k_{i a}\right)$.

Having performed this further decomposition the field strengths and covariant derivatives may be written as

$$
U^{\kappa}=F^{\kappa}+\left(\tilde{\xi}^{\kappa}+M_{0 a}{ }^{\kappa} a^{a}-M_{0}^{a \kappa} b_{a}\right) F^{0}+M_{i a}{ }^{\kappa} a^{a} F^{i}-M_{i}^{a \kappa} b_{a} F^{i},
$$




$$
\begin{aligned}
F^{\kappa} & =d A^{\kappa}+\frac{1}{2}\left(\tilde{k}_{j}^{a} M_{i a}{ }^{\kappa}+k_{j a} M_{i}{ }^{a \kappa}\right) A^{i} \wedge A^{j} \\
\hat{D} \rho_{\alpha} & =D \rho_{\alpha}+\frac{1}{2} M_{\alpha a}{ }^{b}\left(a^{a} D b_{b}-b_{b} D a^{a}\right)-\frac{1}{2} M_{\alpha a b} a^{a} D a^{b}-\frac{1}{2} M_{\alpha}{ }^{a b} b_{a} D b_{b}, \\
D \rho_{\alpha} & =d \rho_{\alpha}-k_{\alpha \kappa} A^{\kappa}+\frac{1}{2} M_{\alpha a}{ }^{b}\left(k_{i b} a^{a}-\tilde{k}_{i}^{a} b_{b}\right) A^{i} .
\end{aligned}
$$

From these expressions we clearly see that a non-Abelian gauge symmetry has emerged after the dualisation. In particular, only the field strength $F^{\kappa}$ includes the usual nonAbelian term $A^{i} \wedge A^{j}$ so that together $\left\{F^{i}, F^{\kappa}\right\}$ correspond to the field strengths of the extended Heisenberg algebra.

To close this section let us discuss the gauge coupling function in some detail. As already mentioned, the Heisenberg group is both non-compact and non-semisimple so the kinetic terms for the gauge bosons cannot be proportional to the Killing form. From (5.16) and using the definition for $U^{\kappa}$ in (5.24) we can read off these kinetic terms as

$$
\begin{aligned}
G^{-1}{ }_{\kappa \lambda} U^{\kappa} \wedge * U^{\lambda}+\tilde{G}_{i j} F^{i} \wedge & * F^{j}=G^{-1}{ }_{\kappa \lambda} F^{\kappa} \wedge * F^{\lambda}+2 G^{-1}{ }_{\kappa \lambda}\left(M_{i a}{ }^{\kappa} a^{a}-M_{i}{ }^{a \kappa} b_{a}\right) F^{\lambda} \wedge * F^{i} \\
& \left(G^{-1}{ }_{\kappa \lambda}\left(M_{i a}{ }^{\kappa} a^{a}-M_{i}{ }^{a \kappa} b_{a}\right)\left(M_{j a}{ }^{\lambda} a^{a}-M_{j}{ }^{a \lambda} b_{a}\right)+\tilde{G}_{i j}\right) F^{i} \wedge * F^{j},
\end{aligned}
$$

where here we have set $A^{0}$ to zero in order to focus only on a particular set of terms. The gauge kinetic function that we see here is independent of the gaugings that we have introduced so corresponds to the supersymmetric result that is also present in the Calabi-Yau fourfold reduction. We also note that it contains the scalars $a^{a}$ and $b_{a}$ in a way that causes it to transform under the gauge symmetries. It is then clear that the constraints (5.14) are needed in order to ensure that the transformation of $a^{a}$ and $b_{a}$ in the gauge kinetic function cancels the variation of $F^{i}$ and $F^{\kappa}$ and so leaves these terms invariant. When the F-theory limit is taken and these kinetic terms are lifted to the corresponding four-dimensional effective theory, this property must be preserved. In addition to this the the gauge kinetic function must become a holomorphic function of the complex coordinates, in order for the action to be supersymmetric.

\section{Conclusions}

In this paper we discussed the appearance of discrete non-Abelian gauge symmetries in Type IIB compactifications to four space-time dimensions. We first reviewed the relationship between discrete symmetries and the gaugings of the isometries of the scalar manifold. We then analysed the symmetries in weakly coupled Type IIB orientifold compactifications that are captured by a generalisation of the Heisenberg algebra. We turned to the gaugings and argued that, when including a D7-brane sector, it appears impossible to find non-Abelian discrete symmetries at weak string coupling. In orientifold reductions with torsion homology we argued that non-Abelian discrete symmetries appear to be in tension with simple supersymmetry considerations. Having carried this out we suggested a concrete scenario which demonstrated that non-Abelian discrete symmetries can arise in more general F-theory compactifications with mutually non-local seven-branes. Remarkably, these considerations require the use of the full power of F-theory away from the weak coupling 
limit. We argued that the gauge fields on general $(p, q)$-seven-branes can gauge both $\mathrm{R}-\mathrm{R}$ and NS-NS axions yielding a non-Abelian gauge structure generalising the Heisenberg algebra. The sources of these gaugings where identified to be: (1) geometric Stückelberg terms on $(p, q)$-seven-branes, (2) fluxes on seven-branes, (3) torsion three-form cohomology in the six-dimensional compactification space $\mathcal{B}_{3}$. It was a non-trivial task to confirm these statements using the duality between M-theory and F-theory. Importantly this required the dualisation of an Abelian theory into a non-Abelian theory in three space-time dimensions.

We have argued that there is a setting in which all fields associated with the gaugings we described arise from seven-branes. To make this more precise one can follow the strategy of $[24,25]$. In these works it was suggested that for Abelian groups the degrees of freedom in the non-linearly charged $N^{a}$ can be captured by open string degrees of freedom $\phi$ linearly charged under the Abelian group. It is natural to conjecture that one can proceed analogously for the non-Abelian configurations considered in this work. For the Heisenberg group such linearly charged states $\phi$ are, for example, given by the theta representation. However, note that these representations of the continuous Heisenberg group are infinite dimensional. At first, this appears to be at odds with the interpretation of $\phi$ as a matter state on intersecting seven-branes. However, the theta representations of the discrete nonAbelian group can be finite dimensional. Recalling that we have found that there is no vacuum of our theory in which the continuous symmetry is unbroken it might therefore be the case that geometrically only the discrete non-Abelian group is realised. Our analysis suggests that it might be possible to find geometries with intersecting seven-branes that have matter linearly charged under a discrete Heisenberg group [36, 54]. The non-Abelian nature of the gaugings then might be tied to the requirement that string junctions between certain seven-branes, as for example (1,0)- and (0,1)-branes, have to exist and end on a third seven-brane. We leave a deeper investigation of such seven-brane settings to future work.

It is interesting to summarise the complications that we had to face in our analysis. Firstly, one could have thought that a straightforward generalisation of the reductions with torsion homology [36] leads to Calabi-Yau fourfold reductions with the desired non-Abelian structure. However, an explicit computation shows that this is not the case. More precisely, a direct reduction of eleven-dimensional supergravity formulated with the three-form field yields only Abelian gaugings even when including torsional cohomology. The non-trivial observation is, however, that this direct reduction is not yet in the correct duality frame to perform the lift to F-theory. After performing the duality, non-Abelian gaugings arise and allow us to identify genuine F-theory gaugings in settings with $(p, q)$-seven-branes. Secondly, showing consistency with supersymmetry turned out to be a non-trivial task which we will to return to. Indeed, in the Type IIB analysis of section 3.2 we found that the reduction considered is not supersymmetric. The local form of the $\mathcal{N}=1$ complex moduli space arising in a general F-theory setting dictates constraints on the allowed holomorphic gaugings.

Let us close by highlighting the intriguing observation we made concerning the gauge coupling functions in the considered F-theory effective actions. If one is able to gauge a noncompact and non-semisimple non-Abelian group, such as the extensions of the Heisenberg groups we found in our settings, then one necessarily has to have a gauge coupling function depending on the complex scalar fields that are charged. In fact, this dependence will by 
partly dictated by the gauge invariance of the action. We have shown that this consistency requirement is automatically satisfied for the F-theory settings we considered. Interestingly, in our setups the modifications of the gauge coupling function are independent of the parameters determining the subgroup of the isometry group that is gauged. One can thus infer properties of the gauge coupling functions in this F-theory reduction by analysing the isometries of the scalar manifold. We have checked that the required modifications give precisely the kinetic mixing terms in standard Type IIB reduction with D7-branes. It would be interesting to understand if this link between holomorphic isometries and the form of the gauge coupling function is a general feature of string theory effective actions.

\section{Acknowledgments}

We are grateful to Sebastian Greiner, Olaf Hohm, Jan Keitel, Fernando Marchesano and especially Iñaki García-Etxebarria for illuminating discussions. This work was supported by a grant from the Max Planck Society.

\section{A Dualisation of the three-dimensional action}

We wish to perform the dualisation of the action (5.7) and to put the fields of the action into a frame that is appropriate for the F-theory lift. In order to simplify the analysis we will first freeze out the moduli dependence of $G_{\Sigma \Lambda}, \tilde{G}_{I J}, G^{I J}$ and $H_{I}{ }^{J}$. We will also make use of (5.10) to remove $N_{\Sigma \Lambda} I$ and $N_{\Sigma \Lambda I}$ from the action. We will then split the index on each field such that those that are to be dualised are identified from those which are not. This will be carried out by splitting

$$
L^{\Sigma}=\left(L^{\hat{\imath}}, L^{\alpha}\right), \quad A^{\Sigma}=\left(A^{\hat{\imath}}, A^{\alpha}\right), \quad \tilde{\xi}^{I}=\left(a^{a}, \tilde{\xi}^{\kappa}\right), \quad \xi_{I}=\left(-b_{a}, \xi_{\kappa}\right) .
$$

where the fields $\xi_{\kappa}$ and $A^{\alpha}$ are to be dualised. With this splitting we will also restrict the gaugings as shown in (5.13) and (5.14) so that the covariant derivatives and field strengths are given by

$$
\begin{aligned}
D a^{a} & =d a^{a}-\tilde{k}_{\hat{\imath}}^{a} A^{\hat{\imath}}, & D b_{a} & =d b_{a}+k_{\hat{\imath} a} A^{\hat{\imath}}, \\
D \tilde{\xi}^{\kappa} & =d \xi^{\kappa}, & D \xi_{\kappa} & =d \xi_{\kappa}-k_{\alpha \kappa} A^{\alpha}, \\
F^{\hat{\imath}} & =d A^{\hat{\imath}}, & F^{\alpha} & =d A^{\alpha},
\end{aligned}
$$

and will restrict $M_{\Sigma I}^{J}$ as shown in (5.15). Performing these steps gives the starting action on which we will perform the duality, given by

$$
\begin{aligned}
S= & \frac{1}{2} \int\left[R * 1-\frac{1}{2} G_{\hat{\imath} \hat{\jmath}} d L^{\hat{\imath}} \wedge * d L^{\hat{\jmath}}-\frac{1}{2} G_{\alpha \beta} d L^{\alpha} \wedge * d L^{\beta}-G_{\hat{\imath} \alpha} d L^{\hat{\imath}} \wedge * d L^{\alpha}\right. \\
& +H_{a}{ }^{b} D a^{a} \wedge * D b_{b}-\tilde{G}_{a \kappa} D a^{a} \wedge * D \tilde{\xi}^{\kappa}-H_{a}{ }^{\kappa} D a^{a} \wedge * D \xi_{\kappa}+H_{\kappa}{ }^{a} D b_{a} \wedge * D \tilde{\xi}^{\kappa} \\
& -H_{\lambda}{ }^{\kappa} D \xi_{\kappa} \wedge * D \tilde{\xi}^{\lambda}-\frac{1}{2} \tilde{G}_{a b} D a^{a} \wedge * D a^{b}-\frac{1}{2} G^{a b} D b_{a} \wedge * D b_{b}-\frac{1}{2} \tilde{G}_{\kappa \lambda} D \tilde{\xi}^{\kappa} \wedge * D \tilde{\xi}^{\lambda} \\
& -\frac{1}{2} G^{\kappa \lambda} D \xi_{\kappa} \wedge * D \xi_{\lambda}-G_{\alpha \hat{\imath}} F^{\alpha} \wedge * F^{\hat{\imath}}+\frac{1}{3} M_{\alpha a}{ }^{b} A^{\alpha} \wedge D a^{a} \wedge D b_{b}+G^{a \kappa} D b_{a} \wedge * D \xi_{\kappa}
\end{aligned}
$$




$$
\begin{aligned}
& +\frac{1}{3} M_{\hat{\imath} a}{ }^{b} A^{\hat{\imath}} \wedge D a^{a} \wedge D b_{b}-\frac{1}{3} M_{\hat{\imath} a \kappa} A^{\hat{\imath}} \wedge D a^{a} \wedge D \tilde{\xi}^{\kappa}-\frac{1}{3} M_{\hat{\imath} a}{ }^{\kappa} A^{\hat{\imath}} \wedge D a^{a} \wedge D \xi_{\kappa} \\
& -\frac{1}{3} M_{\hat{\imath} \kappa}{ }^{a} A^{\hat{\imath}} \wedge D b_{a} \wedge D \tilde{\xi}^{\kappa}+\frac{1}{3} M_{\hat{\imath}}{ }^{a \kappa} A^{\hat{\imath}} \wedge D b_{a} \wedge D \xi_{\kappa}+\frac{1}{3} M_{\hat{\imath} \lambda}{ }^{\kappa} A^{\hat{\imath}} \wedge D \xi_{\kappa} \wedge D \tilde{\xi}^{\lambda} \\
& -\frac{1}{2} G_{\alpha \beta} F^{\alpha} \wedge * F^{\beta}-\frac{1}{2} G_{\hat{\imath} \hat{\jmath}} F^{\hat{\imath}} \wedge * F^{\hat{\jmath}}-\frac{1}{3} M_{\alpha a b} a^{a} D a^{b} \wedge F^{\alpha}-\frac{1}{3} M_{\hat{\imath} a b} a^{a} D a^{b} \wedge F^{\hat{\imath}} \\
& +\frac{1}{3} M_{\alpha a}{ }^{b} a^{a} D b_{b} \wedge F^{\alpha}+\frac{1}{3} M_{\hat{\imath} a}{ }^{b} a^{a} D b_{b} \wedge F^{\hat{\imath}}-\frac{1}{3} M_{\hat{\imath} a \kappa} a^{a} D \tilde{\xi}^{\kappa} \wedge F^{\hat{\imath}}-\frac{1}{3} M_{\hat{\imath} a}{ }^{\kappa} a^{a} D \xi_{\kappa} \wedge F^{\hat{\imath}} \\
& -\frac{1}{6} M_{\alpha}^{a b} A^{\alpha} \wedge D b_{a} \wedge D b_{b}-\frac{1}{6} M_{\hat{\imath} a b} A^{\hat{\imath}} \wedge D a^{a} \wedge D a^{b}-\frac{1}{6} M_{\hat{\imath}}^{a b} A^{\hat{\imath}} \wedge D b_{a} \wedge D b_{b} \\
& -\frac{1}{6} M_{\alpha a b} A^{\alpha} \wedge D a^{a} \wedge D a^{b}-\frac{1}{3} M_{\alpha b}{ }^{a} b_{a} D a^{b} \wedge F^{\alpha}-\frac{1}{3} M_{\hat{\imath} b}{ }^{a} b_{a} D a^{b} \wedge F^{\hat{\imath}} \\
& -\frac{1}{3} M_{\hat{\imath}}{ }^{a b} b_{a} D b_{b} \wedge F^{\hat{\imath}}-\frac{1}{3} M_{\hat{\imath} \kappa}{ }^{a} b_{a} D \tilde{\xi}^{\kappa} \wedge F^{\hat{\imath}}+\frac{1}{3} M_{\hat{\imath}}{ }^{a \kappa} b_{a} D \xi_{\kappa} \wedge F^{\hat{\imath}}-\frac{1}{3} M_{\alpha}{ }^{a b} b_{a} D b_{b} \wedge F^{\alpha} \\
& +\frac{1}{3} M_{\hat{\imath} a \kappa} \tilde{\xi}^{\kappa} D a^{a} \wedge F^{\hat{\imath}}+\frac{1}{3} M_{\hat{\imath} \kappa}{ }^{a} \tilde{\xi}^{\kappa} D b_{a} \wedge F^{\hat{\imath}}+\frac{1}{3} k_{\alpha \kappa} M_{\hat{\imath} \lambda}{ }^{\kappa} \tilde{\xi}^{\lambda} A^{\alpha} \wedge F^{\hat{\imath}} \\
& +\frac{1}{3} k_{\alpha \kappa} M_{\hat{\imath} \lambda}{ }^{\kappa} \tilde{\xi}^{\lambda} A^{\hat{\imath}} \wedge F^{\alpha}-\frac{1}{3} M_{\hat{\imath} \lambda}{ }^{\kappa} \tilde{\xi}^{\lambda} D \xi_{\kappa} \wedge F^{\hat{\imath}}+\frac{1}{3} M_{\hat{\imath} a}{ }^{\kappa} \xi_{\kappa} D a^{a} \wedge F^{\hat{\imath}} \\
& \left.-\frac{1}{3} M_{\hat{\imath}}{ }^{a \kappa} \xi_{\kappa} D b_{a} \wedge F^{\hat{\imath}}+\frac{1}{3} M_{\hat{\imath} \kappa}{ }^{\lambda} \xi_{\lambda} D \tilde{\xi}^{\kappa} \wedge F^{\hat{\imath}}+V * 1\right] .
\end{aligned}
$$

This action has a purely Abelian set of gauge symmetries.

Next let us define the projectors $\Pi_{\alpha}^{\beta}$ and $\Pi_{\lambda}^{\kappa}$ which allow us to identify the fields that participate in the gaugings. These satisfy

$$
\begin{aligned}
\Pi_{\alpha}^{\beta} k_{\beta \kappa} & =k_{\alpha \kappa}, & k_{\alpha \lambda} \Pi_{\kappa}^{\lambda} & =k_{\alpha \kappa}, \\
\Pi_{\alpha}^{\gamma} \Pi_{\gamma}^{\beta} & =\Pi_{\alpha}^{\beta}, & \Pi_{\delta}^{\kappa} \Pi_{\lambda}^{\delta} & =\Pi_{\lambda}^{\kappa},
\end{aligned}
$$

and may be constructed using the so-called Moore-Penrose pseudo-inverse of the matrix $k_{\alpha \kappa}$ which we denote by $\check{k}^{\kappa \alpha}$. Then,

$$
\Pi_{\alpha}^{\beta}=k_{\alpha \kappa} \check{k}^{\kappa \beta}, \quad \quad \Pi_{\kappa}^{\lambda}=\check{k}^{\kappa \alpha} k_{\alpha \lambda} .
$$

In addition to these constraints we will also demand that the projectors satisfy certain symmetry conditions such that

$$
\Pi_{\alpha}^{\gamma} G_{\gamma \beta}=\Pi_{\beta}^{\gamma} G_{\gamma \alpha}, \quad \Pi_{\eta}^{\kappa} G^{\eta \lambda}=\Pi_{\eta}^{\lambda} G^{\eta \kappa} .
$$

These conditions make the pseudo-inverse $\check{k}^{\kappa \alpha}$ unique for a given $k_{\alpha \kappa}$. For convenience we will also define the projectors in the orthogonal directions given by

$$
\Pi_{\perp}^{\beta}{ }_{\alpha}^{\beta}=\left(\delta_{\alpha}^{\beta}-\Pi_{\alpha}^{\beta}\right), \quad \quad \Pi_{\perp}^{\lambda}=\left(\delta_{\kappa}^{\lambda}-\Pi_{\kappa}^{\lambda}\right) .
$$

Having defined these quantities we are now in a position to propose the parent action, from which we will deduce the dual. This is given by,

$$
\begin{aligned}
S= & \frac{1}{2} \int\left[R * 1-\frac{1}{2} G_{\hat{\imath} \hat{\jmath}} d L^{\hat{\imath}} \wedge * d L^{\hat{\jmath}}-\frac{1}{2} G_{\alpha \beta} d L^{\alpha} \wedge * d L^{\beta}-G_{\hat{\imath} \alpha} d L^{\hat{\imath}} \wedge * d L^{\alpha}\right. \\
& +\left(G^{-1}{ }_{\eta \sigma} H_{a}{ }^{\lambda} H_{\kappa}{ }^{\sigma} H_{a}{ }^{b}-G^{b \lambda} G^{-1}{ }_{\kappa \eta} H_{a}{ }^{\kappa}\right) \Pi_{\perp \lambda}{ }_{\lambda}^{\eta} D a^{a} \wedge * D b_{b}-H_{a}{ }^{\lambda} \Pi_{\lambda}^{\kappa} D a^{a} \wedge * D \xi_{\kappa}
\end{aligned}
$$




$$
\begin{aligned}
& +\left(G^{-1}{ }_{\lambda \rho} H_{a}{ }^{\lambda} H_{\kappa}{ }^{\eta} \Pi_{\perp}^{\rho}-\tilde{G}_{a \kappa}\right) D a^{a} \wedge * D \tilde{\xi}^{\kappa} \\
& -G^{a \eta} G^{-1}{ }_{\eta \sigma} H_{\kappa}{ }^{\lambda} \Pi_{\perp \lambda}{ }_{\lambda}^{\sigma} D b_{a} \wedge * D \tilde{\xi}^{\kappa}+H_{\kappa}{ }^{a} D b_{a} \wedge * D \tilde{\xi}^{\kappa}+G^{a \kappa} D b_{a} \wedge * D \xi_{\kappa} \\
& -G^{a \lambda} \Pi_{\perp \lambda}^{\kappa} D b_{a} \wedge * D \xi_{\kappa}+H_{\lambda}{ }^{\eta} \Pi_{\perp}{ }_{\eta}^{\kappa} D \xi_{\kappa} \wedge * D \tilde{\xi}^{\lambda}-H_{\lambda}{ }^{\kappa} D \xi_{\kappa} \wedge * D \tilde{\xi}^{\lambda} \\
& -G_{\beta \gamma} \Pi_{\perp}{ }^{\alpha \gamma} M_{\alpha b}{ }^{a} b_{a} D a^{b} \wedge F^{\beta}+\frac{1}{2} G^{-1}{ }_{\kappa \eta} H_{a}{ }^{\kappa} H_{b}{ }^{\lambda} \Pi_{\perp \lambda}{ }_{\lambda}^{\eta} D a^{a} \wedge * D a^{b} \\
& -\frac{1}{2} \tilde{G}_{a b} D a^{a} \wedge * D a^{b}+G^{-1}{ }_{\eta \rho} H_{a}{ }^{\kappa} \Pi_{\perp}{ }_{\llcorner}^{\eta} D a^{a} \wedge U^{\rho}-\frac{1}{2} G^{a b} D b_{a} \wedge * D b_{b} \\
& +\frac{1}{2} D b_{a} \wedge * D b_{b} G^{a \kappa} G^{b \lambda} G^{-1}{ }_{\kappa \eta} \Pi_{\perp \lambda}^{\eta}-D b_{a} G^{a \kappa} G^{-1}{ }_{\eta \rho} P_{\kappa}^{\eta} \wedge U^{\rho} \\
& +\frac{1}{2} D \tilde{\xi}^{\kappa} \wedge * D \tilde{\xi}^{\lambda} G^{-1}{ }_{\eta \sigma} H_{\kappa}{ }^{\eta} H_{\lambda}{ }^{\rho} \Pi_{\perp}^{\sigma}{ }_{\rho}^{\sigma}-\frac{1}{2} D \tilde{\xi}^{\kappa} \wedge * D \tilde{\xi}^{\lambda} \tilde{G}_{\kappa \lambda} \\
& +D \tilde{\xi}^{\kappa} G_{\lambda \eta}^{-1} H_{\kappa}{ }^{\lambda} \Pi_{\perp}^{\eta} \wedge U^{\sigma}-\frac{1}{2} G^{\kappa \rho} \Pi_{\rho}^{\lambda} D \xi_{\kappa} \wedge * D \xi_{\lambda}+D \xi_{\kappa} \Pi_{\perp_{\eta}}^{\kappa} \wedge U^{\eta}-D \xi_{\kappa} \Pi_{\eta}^{\kappa} \wedge U^{\eta} \\
& -\hat{D} \tilde{\rho}_{\alpha} \Pi_{\perp \beta}^{\alpha} \wedge F^{\beta}-\hat{D} \tilde{\rho}_{\alpha} G_{\beta \hat{\imath}} G^{-1 \alpha \beta} \wedge F^{\hat{\imath}}-\frac{1}{2} G^{-1 \alpha \beta} \hat{D} \tilde{\rho}_{\alpha} \wedge * \hat{D} \tilde{\rho}_{\beta} \\
& +\Pi_{\perp \lambda}^{\eta} M_{\hat{\imath} \kappa}{ }^{\lambda} \xi_{\eta} D \tilde{\xi}^{\kappa} \wedge F^{\hat{\imath}}-\Pi_{\perp}^{\kappa} M_{\hat{\imath}}^{a \lambda} \xi_{\kappa} D b_{a} \wedge F^{\hat{\imath}}+\Pi_{\perp}{ }^{\lambda} M_{\hat{\imath} a}{ }^{\kappa} \xi_{\lambda} D a^{a} \wedge F^{\hat{\imath}} \\
& +\frac{1}{3} M_{\hat{\imath} a}{ }^{b} A^{\hat{\imath}} \wedge D a^{a} \wedge D b_{b}-\frac{1}{3} M_{\hat{\imath} a \kappa} A^{\hat{\imath}} \wedge D a^{a} \wedge D \tilde{\xi}^{\kappa}-\frac{1}{3} M_{\hat{\imath} \kappa}{ }^{a} A^{\hat{\imath}} \wedge D b_{a} \wedge D \tilde{\xi}^{\kappa} \\
& -\frac{1}{2} \Pi_{\perp}^{\beta}{ }_{\alpha}^{\beta} M_{\beta a b} a^{a} D a^{b} \wedge F^{\alpha}-\frac{1}{2} \Pi_{\perp}^{\beta}{ }_{\alpha}^{\beta} M_{\beta}{ }^{a b} b_{a} D b_{b} \wedge F^{\alpha} \\
& -\frac{1}{2} \Pi_{\perp \lambda}^{\eta} G^{-1}{ }_{\eta \rho} U^{\lambda} \wedge * U^{\rho}+\frac{1}{2} G_{\alpha \hat{\imath}} G_{\beta \hat{\jmath}} G^{-1 \alpha \beta} F^{\hat{\imath}} \wedge * F^{\hat{\jmath}}-\frac{1}{2} G_{\hat{\imath} \hat{\jmath}} F^{\hat{\imath}} \wedge * F^{\hat{\jmath}}-\frac{1}{3} M_{\hat{\imath} a b} a^{a} D a^{b} \wedge F^{\hat{\imath}} \\
& +\frac{1}{3} M_{\hat{\imath} a}{ }^{b} a^{a} D b_{b} \wedge F^{\hat{\imath}}-\frac{1}{3} M_{\hat{\imath} a \kappa} a^{a} D \tilde{\xi}^{\kappa} \wedge F^{\hat{\imath}}-\frac{1}{6} M_{\hat{\imath} a b} A^{\hat{\imath}} \wedge D a^{a} \wedge D a^{b} \\
& -\frac{1}{6} M_{\hat{\imath}}{ }^{a b} A^{\hat{\imath}} \wedge D b_{a} \wedge D b_{b}-\frac{1}{3} M_{\hat{\imath} b}{ }^{a} b_{a} D a^{b} \wedge F^{\hat{\imath}}-\frac{1}{3} M_{\hat{\imath}}{ }^{a b} b_{a} D b_{b} \wedge F^{\hat{\imath}} \\
& \left.-\frac{1}{3} M_{\hat{\imath} \kappa}{ }^{a} b_{a} D \tilde{\xi}^{\kappa} \wedge F^{\hat{\imath}}+\frac{1}{3} M_{\hat{\imath} a \kappa} \tilde{\xi}^{\kappa} D a^{a} \wedge F^{\hat{\imath}}+\frac{1}{3} M_{\hat{\imath} \kappa}{ }^{a} \tilde{\xi}^{\kappa} D b_{a} \wedge F^{\hat{\imath}}\right] .
\end{aligned}
$$

In this action the quantities $U^{\kappa}$ and $\hat{D} \rho_{\alpha}$ are not a priori field strengths and covariant derivatives but are instead given by

$$
\begin{aligned}
U^{\kappa}= & \Pi_{\lambda}^{\kappa} d B^{\lambda}+\Pi_{\perp \lambda}^{\kappa} H^{\lambda}+\frac{1}{2} M_{\hat{\imath} a}{ }^{\kappa} F^{\hat{\imath}} a^{a}+\frac{1}{2} M_{\hat{\imath} a}{ }^{\kappa} A^{\hat{\imath}} D a^{a} \\
& +\frac{1}{2} M_{i}{ }^{a \kappa} F^{i} b_{a}+\frac{1}{2} M_{\hat{\imath}}{ }^{\alpha \kappa} A^{\hat{\imath}} D b_{a}+M_{\hat{\imath} \lambda}{ }^{\kappa} F^{\hat{\imath}} \tilde{\xi}^{\lambda}, \\
\hat{D} \rho_{\alpha}= & \Pi_{\alpha}^{\beta} d \rho_{\beta}+\Pi_{\perp}{ }_{\alpha}^{\beta} h_{\beta}-\frac{1}{2} k_{\alpha \kappa} B^{\kappa}-\frac{1}{2} M_{\beta a b} a^{a} D a^{b}+\frac{1}{2} M_{\alpha a}{ }^{b} a^{a} D b_{b} \\
& \left.-\frac{1}{2} M_{\alpha a}{ }^{b} b_{b} D a^{a}-\frac{1}{2} M_{\alpha}{ }^{a b} b_{a} D b_{b}\right),
\end{aligned}
$$

where the fundamental variables in (A.8) are treated as being the variables of (A.3) as well as $B^{\kappa}, H^{\kappa}, \rho_{\beta}$ and $h_{\beta}$.

To verify that the parent Lagrangian (A.8) is indeed equivalent to the starting Lagrangian (A.3) we perform the variation with respect to dual fields that we have introduced. Varying with respect to $B^{\kappa}$ and $H^{\kappa}$ we find that

$$
\begin{aligned}
& U^{\kappa}+\frac{1}{2} D a^{a} \wedge D a^{b} \check{k}^{\kappa \alpha} M_{\alpha a b}-D a^{a} \wedge D b_{b} \check{k}^{\kappa \alpha} M_{\alpha a}{ }^{b}+\frac{1}{2} D b_{a} \wedge D b_{b} \check{k}^{\kappa \alpha} M_{\alpha}^{a b} \\
& -* D \xi_{\eta} G^{\eta \lambda} \Pi_{\perp \lambda}^{\kappa}-* D a^{a} H_{a}{ }^{\lambda} \Pi_{\perp \lambda}^{\kappa}+* D b_{a} G^{a \lambda} \Pi_{\perp \lambda}^{\kappa}-* D \tilde{\xi}^{\eta} H_{\eta}{ }^{\lambda} \Pi_{\perp \lambda}^{\kappa}
\end{aligned}
$$




$$
-\tilde{\xi}^{\eta} \Pi_{\lambda}^{\kappa} F^{\hat{\imath}} M_{\hat{\imath} \eta}{ }^{\lambda}-G_{\alpha \beta} \check{k}^{\kappa \alpha} d * F^{\beta}-G_{\alpha \hat{\imath}} \check{k}^{\kappa \alpha} d * F^{\hat{\imath}}=0,
$$

where the $\Pi_{\lambda}^{\kappa}$ projection of this equation is obtained from the variation with respect to $B^{\kappa}$ and the $\Pi_{\perp}{ }_{\lambda}^{\kappa}$ projection is obtained from the variation with respect to $H^{\kappa}$. Similarly varying with respect to $\rho_{\beta}$ and $h_{\beta}$ gives

$$
\hat{D} \tilde{\rho}_{\alpha}+G_{\alpha \beta} * F^{\beta}+G_{\alpha \hat{\imath}} * F^{\hat{\imath}}=0
$$

where the $\Pi_{\alpha}^{\beta}$ projection comes from the variation with respect to $\rho_{\alpha}$ and the $\Pi_{\perp}^{\beta}$ projection comes from the variation with respect to $h_{\beta}$.

Substituting these equations into (A.8) and making use of certain total derivative identities we return to the original Lagrangian (A.3). This identifies that the Lagrangian (A.8) represents an appropriate parent Lagrangian with which to perform the dualisation of (A.3).

Next we may consider varying the action (A.8) with respect to the old variables $\xi_{\kappa}$ and $A^{\alpha}$. The variation with respect to $A^{\alpha}$ is most easily understood by splitting it into its $\Pi_{\alpha}^{\beta}$ and $\Pi_{\perp}^{\beta}$ projections. The $\Pi_{\alpha}^{\beta}$ projection contracted with $\check{k}^{\alpha \kappa}$ gives

$$
D \xi_{\eta} G^{\eta \lambda} \Pi_{\lambda}^{\kappa}+D \tilde{\xi}^{\eta} H_{\eta}{ }^{\lambda} \Pi_{\lambda}^{\kappa}+D a^{a} H_{a}{ }^{\lambda} \Pi_{\lambda}^{\kappa}-D b_{a} G^{a \lambda} \Pi_{\lambda}^{\kappa}+\Pi_{\lambda}^{\kappa} * U^{\lambda}=0,
$$

while the $\Pi_{\perp}^{\beta}$ projection gives a Bianchi identity for $h_{\beta}$ which implies that

$$
\begin{aligned}
\hat{D} \rho_{\alpha}= & d \rho_{\beta}-\frac{1}{2} k_{\beta \kappa} B^{\kappa}-\frac{1}{2} M_{\alpha a b} a^{a} D a^{b}+\frac{1}{2} M_{\alpha a}{ }^{b} a^{a} D b_{b} \\
& -\frac{1}{2} M_{\alpha a}{ }^{b} b_{b} D a^{a}-\frac{1}{2} M_{\alpha}^{a b} b_{a} D b_{b}
\end{aligned}
$$

Similarly the variation of (A.8) with respect to $\xi_{\kappa}$ is most easily understood by considering its projections with respect to $\Pi_{\lambda}^{\kappa}$ and $\Pi_{\perp \lambda}^{\kappa}$. The $\Pi_{\lambda}^{\kappa}$ projection gives an equation which represents the derivative of (A.12) so imposes no additional constraint. Alternatively, the $\Pi_{\perp}^{\kappa}$ projection implies a Bianchi identity for $H^{\kappa}$ which is solved by

$$
\begin{aligned}
U^{\kappa}= & d B^{\lambda}+\frac{1}{2} M_{\hat{\imath} a}{ }^{\kappa} F^{\hat{\imath}} a^{a}+\frac{1}{2} M_{\hat{\imath} a}{ }^{\kappa} A^{\hat{\imath}} D a^{a} \\
& +\frac{1}{2} M_{\hat{\imath}}{ }^{a \kappa} F^{\hat{\imath}} b_{a}+\frac{1}{2} M_{\hat{\imath}}{ }^{\kappa} A^{\hat{\imath}} D b_{a}+M_{\hat{\imath} \lambda}{ }^{\kappa} F^{\hat{\imath}} \tilde{\xi}^{\lambda} .
\end{aligned}
$$

Finally we may form a further useful equation by taking the exterior derivative of (A.12) and contracting with $\breve{k}^{\alpha \kappa}$, which gives

$$
\begin{aligned}
& \Pi_{\beta}^{\alpha} F^{\beta}-G_{\kappa \lambda}^{-1} H_{a}{ }^{\kappa} \check{k}^{\lambda \alpha} d D a^{a}-G^{-1}{ }_{\lambda \eta} H_{\kappa}{ }^{\lambda} \check{k}^{\eta \alpha} d D \tilde{\xi}^{\kappa} \\
& +G^{a \lambda} G^{-1}{ }_{\kappa \lambda} \check{k}^{\kappa \alpha} d D b_{a}-G^{-1}{ }_{\kappa \lambda} \check{k}^{\kappa \alpha} d * U^{\lambda}=0 .
\end{aligned}
$$

Then splitting $D \xi_{\kappa}$ and $F^{\alpha}$ into their two projections in (A.8) we may use (A.12) to eliminate $\Pi_{\kappa}^{\lambda} D \xi_{\lambda}$ and (A.15) to eliminate $\Pi_{\beta}^{\alpha} F^{\beta}$. We may then use (A.13), (A.14) and various total derivative identities to eliminate the remaining projections $\Pi_{\perp}{ }_{k}^{\lambda} D \xi_{\lambda}$ and $\Pi_{\perp \beta}^{\alpha} F^{\beta}$. Having done this we arrive at the dual Lagrangian 


$$
\begin{aligned}
S= & \frac{1}{2} \int\left[R * 1-\frac{1}{2} G_{\hat{\imath} \hat{\jmath}} d L^{\hat{\imath}} \wedge * d L^{\hat{\jmath}}-\frac{1}{2} G_{\alpha \beta} d L^{\alpha} \wedge * d L^{\beta}-G_{\hat{\imath} \alpha} d L^{\hat{\imath}} \wedge * d L^{\alpha}\right. \\
& -\frac{1}{2} \tilde{G}_{a b} D a^{a} \wedge * D a^{b}-\frac{1}{2} \tilde{G}^{a b} D b_{a} \wedge * D b_{b}+\tilde{H}_{a}{ }^{b} D a^{a} \wedge * D b_{b}-\frac{1}{2} G^{-1 \alpha \beta} \hat{D} \rho_{\alpha} \wedge * \hat{D} \rho_{\beta} \\
& -\frac{1}{2} \tilde{G}_{\kappa \lambda} D \tilde{\xi}^{\kappa} \wedge * D \tilde{\xi}^{\lambda}-\tilde{G}_{a \kappa} D a^{a} \wedge * D \tilde{\xi}^{\kappa}+\tilde{H}_{\kappa}{ }^{a} D b_{a} \wedge * D \tilde{\xi}^{\kappa}-G^{-1}{ }_{\kappa \lambda} G^{a \kappa} D b_{a} \wedge U^{\lambda} \\
& +G^{-1}{ }_{\kappa \lambda} H_{a}{ }^{\kappa} D a^{a} \wedge U^{\lambda}+G^{-1} \lambda \eta H_{\kappa}{ }^{\lambda} D \tilde{\xi}^{\kappa} \wedge U^{\eta}-G^{-1 \alpha \beta} G_{\beta \hat{\imath}} \hat{D} \rho_{\alpha} \wedge F^{\hat{\imath}} \\
& -\frac{1}{3} M_{\hat{\imath} a \kappa} A^{\hat{\imath}} \wedge D a^{a} \wedge D \tilde{\xi}^{\kappa}-\frac{1}{3} M_{\hat{\imath} \kappa}{ }^{a} A^{\hat{\imath}} \wedge D b_{a} \wedge D \tilde{\xi}^{\kappa}+\frac{1}{3} M_{\hat{\imath} a}{ }^{b} A^{\hat{\imath}} \wedge D a^{a} \wedge D b_{b} \\
& -\frac{1}{2} G^{-1}{ }_{\kappa \lambda} U^{\kappa} \wedge * U^{\lambda}-\frac{1}{2} G_{\hat{\imath} \hat{\jmath}} F^{\hat{\imath}} \wedge * F^{\hat{\jmath}}-\frac{1}{3} M_{\hat{\imath} a b} a^{a} D a^{b} \wedge F^{\hat{\imath}}+\frac{1}{2} G^{-1 \alpha \beta} G_{\alpha \hat{\imath}} G_{\beta \hat{\jmath}} F^{\hat{\imath}} \wedge * F^{\hat{\jmath}} \\
& +\frac{1}{3} M_{\hat{\imath} a}{ }^{b} a^{a} D b_{b} \wedge F^{\hat{\imath}}-\frac{1}{3} M_{\hat{\imath} a \kappa} a^{a} D \tilde{\xi}^{\kappa} \wedge F^{\hat{\imath}}-\frac{1}{6} M_{\hat{\imath} a b} A^{\hat{\imath}} \wedge D a^{a} \wedge D a^{b} \\
& -\frac{1}{6} M_{\hat{\imath}}^{a b} A^{\hat{\imath}} \wedge D b_{a} \wedge D b_{b}-\frac{1}{3} M_{\hat{\imath} b}{ }^{a} b_{a} D a^{b} \wedge F^{\hat{\imath}}-\frac{1}{3} M_{\hat{\imath}}^{a b} b_{a} D b_{b} \wedge F^{\hat{\imath}} \\
& \left.-\frac{1}{3} M_{\hat{\imath} \kappa}{ }^{a} b_{a} D \tilde{\xi}^{\kappa} \wedge F^{\hat{\imath}}+\frac{1}{3} M_{\hat{\imath} a \kappa} \tilde{\xi}^{\kappa} D a^{a} \wedge F^{\hat{\imath}}+\frac{1}{3} M_{\hat{\imath} \kappa}^{a} \tilde{\xi}^{\kappa} D b_{a} \wedge F^{\hat{\imath}}+V * 1\right] .
\end{aligned}
$$

We may then make the symmetries of (A.17) more transparent by making the field redefinition

$$
B^{\kappa}=A^{\kappa}+\frac{1}{2} M_{\hat{\imath} a}{ }^{\kappa} A^{\hat{\imath}} a^{a}-\frac{1}{2} M_{\hat{\imath}}{ }^{\alpha \kappa} A^{\hat{\imath}} b_{a} .
$$

This allows $U^{\kappa}$ and $\hat{D} \rho_{\alpha}$ to be written as shown in (5.18).

Open Access. This article is distributed under the terms of the Creative Commons Attribution License (CC-BY 4.0), which permits any use, distribution and reproduction in any medium, provided the original author(s) and source are credited.

\section{References}

[1] C. Vafa, Evidence for F-theory, Nucl. Phys. B 469 (1996) 403 [hep-th/9602022] [InSPIRE].

[2] H. Hayashi, C. Lawrie, D.R. Morrison and S. Schäfer-Nameki, Box graphs and singular fibers, JHEP 05 (2014) 048 [arXiv: 1402.2653] [INSPIRE].

[3] A.P. Braun and S. Schäfer-Nameki, Box graphs and resolutions I, arXiv:1407.3520 [INSPIRE].

[4] M. Esole, S.-H. Shao and S.-T. Yau, Singularities and gauge theory phases, arXiv:1402.6331 [INSPIRE].

[5] M. Esole, S.-H. Shao and S.-T. Yau, Singularities and gauge theory phases II, arXiv: 1407.1867 [INSPIRE].

[6] T.W. Grimm and T. Weigand, On Abelian gauge symmetries and proton decay in global F-theory GUTs, Phys. Rev. D 82 (2010) 086009 [arXiv:1006.0226] [InSPIRE].

[7] D.R. Morrison and D.S. Park, F-theory and the Mordell-Weil group of elliptically-fibered Calabi-Yau threefolds, JHEP 10 (2012) 128 [arXiv:1208.2695] [INSPIRE].

[8] V. Braun, T.W. Grimm and J. Keitel, New global F-theory GUTs with U(1) symmetries, JHEP 09 (2013) 154 [arXiv: 1302.1854] [INSPIRE]. 
[9] T.W. Grimm, A. Kapfer and J. Keitel, Effective action of $6 D$ F-theory with U(1) factors: rational sections make Chern-Simons terms jump, JHEP 07 (2013) 115 [arXiv:1305.1929] [INSPIRE].

[10] M. Kuntzler and S. Schäfer-Nameki, Tate trees for elliptic fibrations with rank one Mordell-Weil group, arXiv:1406.5174 [INSPIRE].

[11] J. Borchmann, C. Mayrhofer, E. Palti and T. Weigand, SU(5) tops with multiple U(1)s in F-theory, Nucl. Phys. B 882 (2014) 1 [arXiv:1307.2902] [inSPIRE].

[12] M. Cvetič, D. Klevers and H. Piragua, F-theory compactifications with multiple U(1)-factors: constructing elliptic fibrations with rational sections, JHEP 06 (2013) 067 [arXiv:1303.6970] [INSPIRE].

[13] M. Cvetič, A. Grassi, D. Klevers and H. Piragua, Chiral four-dimensional F-theory compactifications with $\mathrm{SU}(5)$ and multiple $\mathrm{U}(1)$-factors, JHEP 04 (2014) 010 [arXiv: 1306.3987] [INSPIRE].

[14] J. Borchmann, C. Mayrhofer, E. Palti and T. Weigand, Elliptic fibrations for $\mathrm{SU}(5) \times \mathrm{U}(1) \times \mathrm{U}(1)$ F-theory vacua, Phys. Rev. D 88 (2013) 046005 [arXiv: 1303.5054] [INSPIRE].

[15] M. Cvetič, D. Klevers, H. Piragua and P. Song, Elliptic fibrations with rank three Mordell-Weil group: F-theory with $\mathrm{U}(1) \times \mathrm{U}(1) \times \mathrm{U}(1)$ gauge symmetry, JHEP 03 (2014) 021 [arXiv: 1310.0463] [INSPIRE].

[16] V. Braun, T.W. Grimm and J. Keitel, Geometric engineering in toric F-theory and GUTs with U(1) gauge factors, JHEP 12 (2013) 069 [arXiv: 1306.0577] [INSPIRE].

[17] D. Klevers, D.K.M. Peña, P.-K. Oehlmann, H. Piragua and J. Reuter, F-theory on all toric hypersurface fibrations and its Higgs branches, JHEP 01 (2015) 142 [arXiv:1408.4808] [INSPIRE].

[18] V. Braun, T.W. Grimm and J. Keitel, Complete intersection fibers in F-theory, JHEP 03 (2015) 125 [arXiv:1411.2615] [INSPIRE].

[19] C. Lawrie and D. Sacco, Tate's algorithm for F-theory GUTs with two U(1)s, JHEP 03 (2015) 055 [arXiv:1412.4125] [INSPIRE].

[20] C. Lawrie, S. Schäfer-Nameki and J.-M. Wong, F-theory and all things rational: surveying U(1) symmetries with rational sections, JHEP 09 (2015) 144 [arXiv:1504.05593] [INSPIRE].

[21] T.W. Grimm and A. Kapfer, Anomaly cancelation in field theory and F-theory on a circle, arXiv:1502.05398 [INSPIRE].

[22] V. Braun and D.R. Morrison, F-theory on genus-one fibrations, JHEP 08 (2014) 132 [arXiv: 1401.7844] [INSPIRE].

[23] D.R. Morrison and W. Taylor, Sections, multisections and U(1) fields in F-theory, arXiv: 1404.1527 [INSPIRE].

[24] L.B. Anderson, I. García-Etxebarria, T.W. Grimm and J. Keitel, Physics of F-theory compactifications without section, JHEP 12 (2014) 156 [arXiv:1406.5180] [INSPIRE].

[25] I. García-Etxebarria, T.W. Grimm and J. Keitel, Yukawas and discrete symmetries in F-theory compactifications without section, JHEP 11 (2014) 125 [arXiv:1408.6448] [INSPIRE]. 
[26] C. Mayrhofer, E. Palti, O. Till and T. Weigand, Discrete gauge symmetries by Higgsing in four-dimensional F-theory compactifications, JHEP 12 (2014) 068 [arXiv:1408.6831] [INSPIRE].

[27] C. Mayrhofer, E. Palti, O. Till and T. Weigand, On discrete symmetries and torsion homology in F-theory, JHEP 06 (2015) 029 [arXiv: 1410.7814] [INSPIRE].

[28] M. Cvetič, R. Donagi, D. Klevers, H. Piragua and M. Poretschkin, F-theory vacua with $Z_{3}$ gauge symmetry, Nucl. Phys. B 898 (2015) 736 [arXiv:1502.06953] [INSPIRE].

[29] M.R. Douglas, D.S. Park and C. Schnell, The Cremmer-Scherk mechanism in F-theory compactifications on K3 manifolds, JHEP 05 (2014) 135 [arXiv:1403.1595] [INSPIRE].

[30] T. Banks and N. Seiberg, Symmetries and strings in field theory and gravity, Phys. Rev. D 83 (2011) 084019 [arXiv:1011.5120] [INSPIRE].

[31] T.W. Grimm, M. Kerstan, E. Palti and T. Weigand, Massive Abelian gauge symmetries and fluxes in F-theory, JHEP 12 (2011) 004 [arXiv:1107.3842] [INSPIRE].

[32] T.W. Grimm and T.G. Pugh, Gauged supergravities and their symmetry-breaking vacua in F-theory, JHEP 06 (2013) 012 [arXiv: 1302.3223] [INSPIRE].

[33] A.P. Braun, A. Collinucci and R. Valandro, The fate of U(1)'s at strong coupling in F-theory, JHEP 07 (2014) 028 [arXiv:1402.4054] [INSPIRE].

[34] H. Jockers and J. Louis, The effective action of D7-branes in $N=1$ Calabi-Yau orientifolds, Nucl. Phys. B 705 (2005) 167 [hep-th/0409098] [INSPIRE].

[35] P.G. Cámara, L.E. Ibáñez and F. Marchesano, RR photons, JHEP 09 (2011) 110 [arXiv:1106.0060] [INSPIRE].

[36] M. Berasaluce-González, P.G. Cámara, F. Marchesano, D. Regalado and A.M. Uranga, Non-Abelian discrete gauge symmetries in 4d string models, JHEP 09 (2012) 059 [arXiv: 1206.2383] [INSPIRE].

[37] M. Cvetič, D. Klevers, D.K.M. Peña, P.-K. Oehlmann and J. Reuter, Three-family particle physics models from global F-theory compactifications, JHEP 08 (2015) 087 [arXiv: 1503.02068] [INSPIRE].

[38] S. Gukov, M. Rangamani and E. Witten, Dibaryons, strings and branes in AdS orbifold models, JHEP 12 (1998) 025 [hep-th/9811048] [INSPIRE].

[39] M. Berasaluce-González, L.E. Ibáñez, P. Soler and A.M. Uranga, Discrete gauge symmetries in D-brane models, JHEP 12 (2011) 113 [arXiv:1106.4169] [INSPIRE].

[40] C.M. Hull, A. Karlhede, U. Lindström and M. Roček, Nonlinear $\sigma$ models and their gauging in and out of superspace, Nucl. Phys. B 266 (1986) 1 [INSPIRE].

[41] J.A. Harvey, TASI 2003 lectures on anomalies, hep-th/0509097 [INSPIRE].

[42] A. Bilal, Lectures on anomalies, arXiv:0802.0634 [INSPIRE].

[43] T.W. Grimm and J. Louis, The effective action of $N=1$ Calabi-Yau orientifolds, Nucl. Phys. B 699 (2004) 387 [hep-th/0403067] [INSPIRE].

[44] T.W. Grimm, The $N=1$ effective action of F-theory compactifications, Nucl. Phys. B 845 (2011) 48 [arXiv: 1008.4133] [INSPIRE].

[45] M. Berasaluce-González, P.G. Cámara, F. Marchesano and A.M. Uranga, $Z_{p}$ charged branes in flux compactifications, JHEP 04 (2013) 138 [arXiv:1211.5317] [INSPIRE]. 
[46] H. Samtleben, Lectures on gauged supergravity and flux compactifications, Class. Quant. Grav. 25 (2008) 214002 [arXiv:0808.4076] [INSPIRE].

[47] B. de Wit, H. Nicolai and H. Samtleben, Gauged supergravities in three-dimensions: a panoramic overview, PoS (jhw2003) 016 [PoS (jhw2003) 018] [hep-th/0403014] [INSPIRE].

[48] S.A. Abel, M.D. Goodsell, J. Jaeckel, V.V. Khoze and A. Ringwald, Kinetic mixing of the photon with hidden $\mathrm{U}(1)$ s in string phenomenology, JHEP 07 (2008) 124 [arXiv:0803.1449] [INSPIRE].

[49] F. Marchesano, D. Regalado and G. Zoccarato, U(1) mixing and D-brane linear equivalence, JHEP 08 (2014) 157 [arXiv: 1406.2729] [INSPIRE].

[50] T.W. Grimm, D. Regalado and T.G. Pugh, to appear.

[51] T.W. Grimm and R. Savelli, Gravitational instantons and fluxes from $M / F$-theory on Calabi-Yau fourfolds, Phys. Rev. D 85 (2012) 026003 [arXiv:1109.3191] [INSPIRE].

[52] T.W. Grimm and H. Hayashi, F-theory fluxes, chirality and Chern-Simons theories, JHEP 03 (2012) 027 [arXiv:1111.1232] [INSPIRE].

[53] T.W. Grimm and D. Regalado, On dualities for non-Abelian gauge theories with continuous center, arXiv: 1509.04281 [INSPIRE].

[54] F. Marchesano, D. Regalado and L. Vázquez-Mercado, Discrete flavor symmetries in D-brane models, JHEP 09 (2013) 028 [arXiv: 1306.1284] [INSPIRE]. 